University of New Hampshire

University of New Hampshire Scholars' Repository

Physics Scholarship

Physics

7-1986

\title{
The average magnetic field draping and consistent plasma properties of the Venus magnetotail
}

\author{
David J. McComas \\ Los Alamos National Laboratory \\ Harlan E. Spence \\ University of New Hampshire, harlan.spence@unh.edu \\ C. T. Russell \\ University of California - Los Angeles
}

M. A. Saunders

Imperial College of Science and Technology

Follow this and additional works at: https://scholars.unh.edu/physics_facpub

Part of the Physics Commons

\section{Recommended Citation}

McComas, D. J., H. E. Spence, C. T. Russell, and M. A. Saunders (1986), The average magnetic field draping and consistent plasma properties of the Venus magnetotail, J. Geophys. Res., 91(A7), 7939-7953, doi:10.1029/JA091iA07p07939.

This Article is brought to you for free and open access by the Physics at University of New Hampshire Scholars' Repository. It has been accepted for inclusion in Physics Scholarship by an authorized administrator of University of New Hampshire Scholars' Repository. For more information, please contact Scholarly.Communication@unh.edu. 


\title{
The Average Magnetic Field Draping and Consistent Plasma Properties of the Venus Magnetotail
}

\author{
D. J. McComas \\ Los Alamos National Laboratory, Los Alamos, New Mexico \\ H. E. SPENCE AND C. T. RusSell \\ Institute of Geophysics and Planetary Physics, University of California, Los Angeles \\ M. A. SAUNDERS \\ The Blackett Laboratory, Imperial College of Science and Technology, London
}

\begin{abstract}
A new technique has been developed to determine the average structure of the Venus magnetotail (in the range from $-8 R_{V}$ to $-12 R_{V}$ ) from the Pioneer Venus magnetometer observations. The spacecraft position with respect to the cross-tail current sheet is determined from an observed relationship between the field-draping angle and the magnitude of the field referenced to its value in the nearby magnetosheath. This allows us statistically to remove the effects of tail flapping and variability of draping for the first time and thus to map the average field configuration in the Venus tail. From this average configuration we calculate the cross-tail current density distribution and $\mathbf{J} \times \mathbf{B}$ forces. Continuity of the tangential electric field is utilized to determine the average variations of the $X$-directed velocity which is shown to vary from $-250 \mathrm{~km} / \mathrm{s}$ at $-8 R_{V}$ to $-470 \mathrm{~km} / \mathrm{s}$ at $-12 R_{V}$. From the calculated $\mathrm{J} \times$ B forces, plasma velocity, and MHD momentum equation the approximate plasma acceleration, density, and temperature in the Venus tail are determined. The derived ion density is approximately $\sim 0.07 p^{+} / \mathrm{cm}^{3}$ $\left(0.005 \mathrm{O}^{+} / \mathrm{cm}^{3}\right)$ in the lobes and $\sim 0.9 \mathrm{p}^{+} / \mathrm{cm}^{3}\left(0.06 \mathrm{O}^{+} / \mathrm{cm}^{3}\right)$ in the current sheet, while the derived approximate average plasma temperature for the tail is $\sim 6 \times 10^{6} \mathrm{~K}$ for a hydrogen plasma or $\sim 9 \times 10^{7}$ $\mathrm{K}$ for an oxygen plasma.
\end{abstract}

\section{INTRODUCTION}

Extensive in situ observations of the magnetic field and plasma populations in the Venus environs have shown that (1) Venus does not have a significant intrinsic magnetic field [Russell et al., 1980a] and (2) a magnetotail is a regular feature of the region antisunward of the planet [Russell, 1976; Dolginov et al., 1978; Russell et al., 1981, 1985; Intriligator and Scarf, 1984; Slavin et al., 1984; Saunders and Russell, 1986]. The Venus magnetotail is generally believed to form by magnetic field draping about the Venus ionosphere in a manner first suggested to explain comet tails by Alfvén [1957].

A steady state configuration of this interaction is shown schematically in Figure 1. The solar wind, with the imbedded interplanetary magnetic field (IMF), flows radially outward from,the rotating sun, causing the well-known Parker spiral pattern of the IMF. The solar wind carries the IMF flux through Venus's bow shock and mass loading, extended, neutral exosphere, and then past the generally unmagnetized but conducting ionosphere. Little, if any, of the upstream plasma flow enters Venus's ionosphere. Rather, the flow is slowed, compressed, and deflected above the dayside ionopause and eventually slips around the planet. The plasma in the magnetosheath to the sides of the Venus obstacle and magnetotail regions travels at the larger velocity of the magnetosheath flow, which is a function of position behind the shock. Since the magnetic field lines link the magnetosheath and nearVenus regions, the lines become bent. This has the effect of draping the field lines so that they are "hung up" on Venus

Copyright 1986 by the American Geophysical Union.

Paper number 5A8292.

0148-0227/86/00SA-8292\$05.00 and stretched out generally sunward and antisunward to the sides of the Venus magnetotail.

In addition to deflection of the flow by the conductive ionosphere, viscous slowing of the flow by the ionosphere and mass loading of the magnetosheath plasma with material from the extended Venus atmosphere and ionosphere also contribute to Venus magnetotail formation. The mass-loaded plasma flow slows in order to conserve momentum, which significantly enhances the draping of field lines around the conducting ionosphere and substantially helps produce the Venus magnetotail. Eventually, somewhere very far downstream, we expect that the field lines should once again assume their interplanetary configuration owing to the Maxwell stresses in the draped magnetic field configuration. These stresses should cause the kinked portions of the field lines to accelerate back up to, and beyond, the solar wind speed, while mass-loaded material tends to diffuse along the field lines owing to its parallel plasma temperature.

There is now much direct and indirect evidence for mass loading of the Venus magnetosheath by the pickup of newly created ions and other processes, such as charge exchange. Oxygen ions have been seen in the near-terminator region of the Venus magnetosheath [Mihalov and Barnes, 1981] and occasionally in the distant magnetosheath and magnetotail region [Mihalov and Barnes, 1982]. An enhancement of the magnetosheath magnetic field strength has been found in the hemisphere where most ion mass pickup is expected, presumably due to a decrease of the magnetosheath flow speed and the consequent pileup of the plasma density and magnetic field [Luhmann et al., 1985]. Other indirect evidence is that the position of the bow shock at the Venus terminator has been found to depend on solar activity. When the solar EUV flux is 


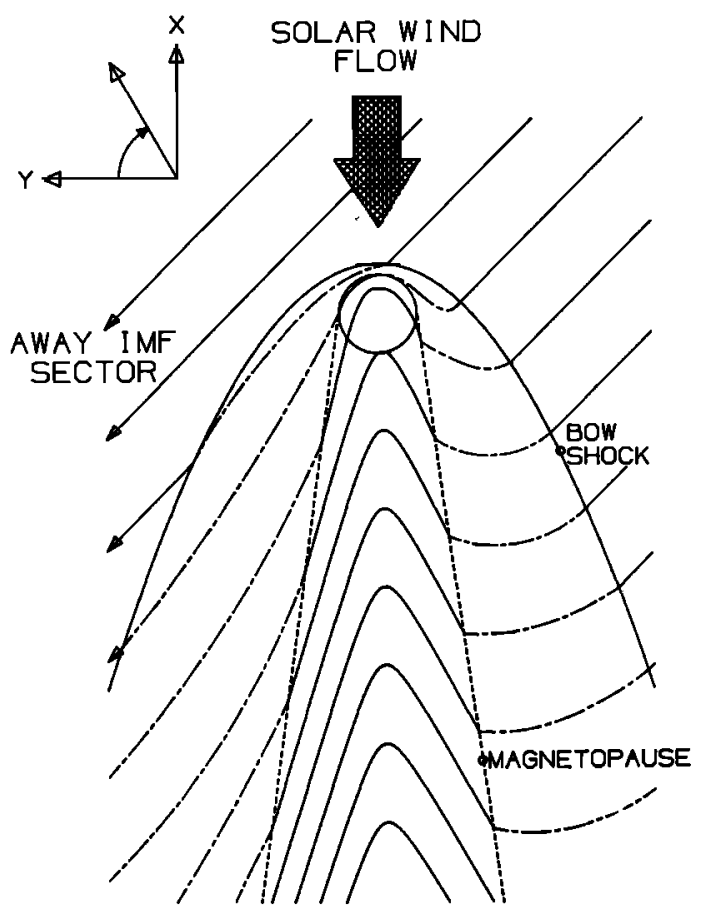

Fig. 1. Schematic diagram of the postulated solar wind interaction with Venus in the plane containing the upstream IMF and solar wind flow vector. The upstream solar wind carries the IMF through the bow shock and magnetosheath. At the obstacle the magnetic field must flow perpendicular to this plane and around the conductive ionosphere. This diversion of the flow, enhanced by mass loading of the flow near to the obstacle, causes the field lines to drape and form a magnetotail. Note also that the spiral orientation of the upstream magnetic field causes the magnetic flux in the left lobe to be greater than in the right at every distance downtail. This has important dynamical implications for the actual tail configuration (see text).

high, it appears that more mass is being added to the shocked solar wind, forcing the bow shock to recede from the planet [Alexander and Russell, 1985].

An interesting asymmetry between the two draped lobes is observed in Figure 1. If this schematic is correct, the $X$ component of the IMF has an important impact on the internal magnetic configuration of the Venus magnetotail. As a consequence of the larger magnetic flux content of the tailward pointing lobe at all distances down the tail, the current sheet should be displaced to the right in an equilibrium configuration. As the IMF spiral angle (in the $X-Y$ plane) rotates, the current sheet should tend to flap from side to side within the tail in order to try to maintain an equilibrium condition. In this study we choose a coordinate system which preserves any asymmetries between the lobes and examine the importance of this effect in the morphology of the Venus magnetotail.

The purpose of this paper is to examine the detailed average draping pattern of the magnetic field in the deep $(-8$ to -12 $R_{V}$ ) Venus magnetotail. A detailed mapping of the average configuration of this region is made possible for the first time by a new technique. This technique removes the ambiguity of the spacecraft location with respect to the tail structures by utilizing an observed relationship between the magnetic field angle and the field magnitude referenced to the immediately adjacent magnetosheath value, or diamagnetic reduction. A statistical study of the field variations with respect to the internal tail structure is thereby made possible, and the average plasma properties consistent with these variations are derived.

We will describe this study in sections. Section 2 examines the variability of the data ordered by spatial location and lays the groundwork for developing a coordinate system which measures locations with respect to the tail structures themselves. Section 3 shows how we reconstruct the structure of the tail in the presence of flapping and examines the average variations in the field components, culminating in the average field vectors, cross-tail current density distribution, and $\mathbf{J} \times \mathbf{B}$ forces as functions of location across the tail. Section 4 derives the average downtail velocity as a function of distance and defines a simple model based on the field variations from which the average plasma acceleration as a function of distance, density, and temperature are obtained. Finally, the "Summary" reviews the most salient steps and results of our analysis.

\section{The Average Venus Tail in Magnetic COORDINATES}

The data set used in this study is a large subset of the data set chosen by Saunders and Russell [1986]. It consists of magnetic field data from the Pioneer Venus Orbiter (PVO) magnetometer [Russell et al., 1980b] and contains the magnetotail portions of 38 orbits which were selected from the first 10 seasons of data (June 1979 to May 1984). In all, 9423 1-min averaged magnetic field measurements are used. The criteria used to identify magnetotail portions of the data are variations in field strength and orientation, and changes in the spectrum of field variations; they are described in greater detail, with examples, by Saunders and Russell [1986]. While the identification of these regions is not perfect, it is correct for the vast majority of the data, and the mixing in of small portions of magnetosheath data will not substantially affect the statistical results described here.

The coordinate system used in this section of our study will be called the $B-v$ coordinate system. The term $B-v$ is used to indicate that the orientation of the coordinate system is determined by the average upstream magnetic field and solar wind flow directions. In the $B-v$ coordinate system the solar wind flows parallel to the $-X$ direction, and the magnetic field component perpendicular to the solar wind flow lies along the $+Y$ direction. The $B-v$ coordinate system is derived from the aberrated VSO coordinate system, described by Saunders and Russell [1986]. The VSO coordinate system is similar to the GSE coordinate system, except that it is centered on Venus and its pole is parallel to the Venus orbital pole. The VSO $X$ and $Y$ axes are then rotated by $5^{\circ}$ about the $Z$ axis to remove the average aberration of the solar wind flow due to Venus's orbital motion.

In order to convert from an aberrated VSO coordinate system to a magnetic field ordered system we have two choices. The standard choice would be to rotate about the solar wind direction so that the magnetic field projected on the $Y-Z$ plane always pointed in the same direction, e.g., $+Y$ [Bieber and Stone, 1979; Saunders and Russell, 1986]. There is a difliculty with this method, however, for if there is an asymmetry between the two lobes in the tail, as discussed previously, this method would average out the difference by combining instances of each type of lobe on both the $\pm Y$ sides of the tail. The alternative choice is to assume that the sector polarity of the magnetic field is inconsequential to the draping of the field and change the sense of each field line so that it corresponds to a magnetic field line pointing away from the sun. This gives a coordinate system where the field is always rotated by less than $\pm 90^{\circ}$ to project along the $+Y$ direction. 


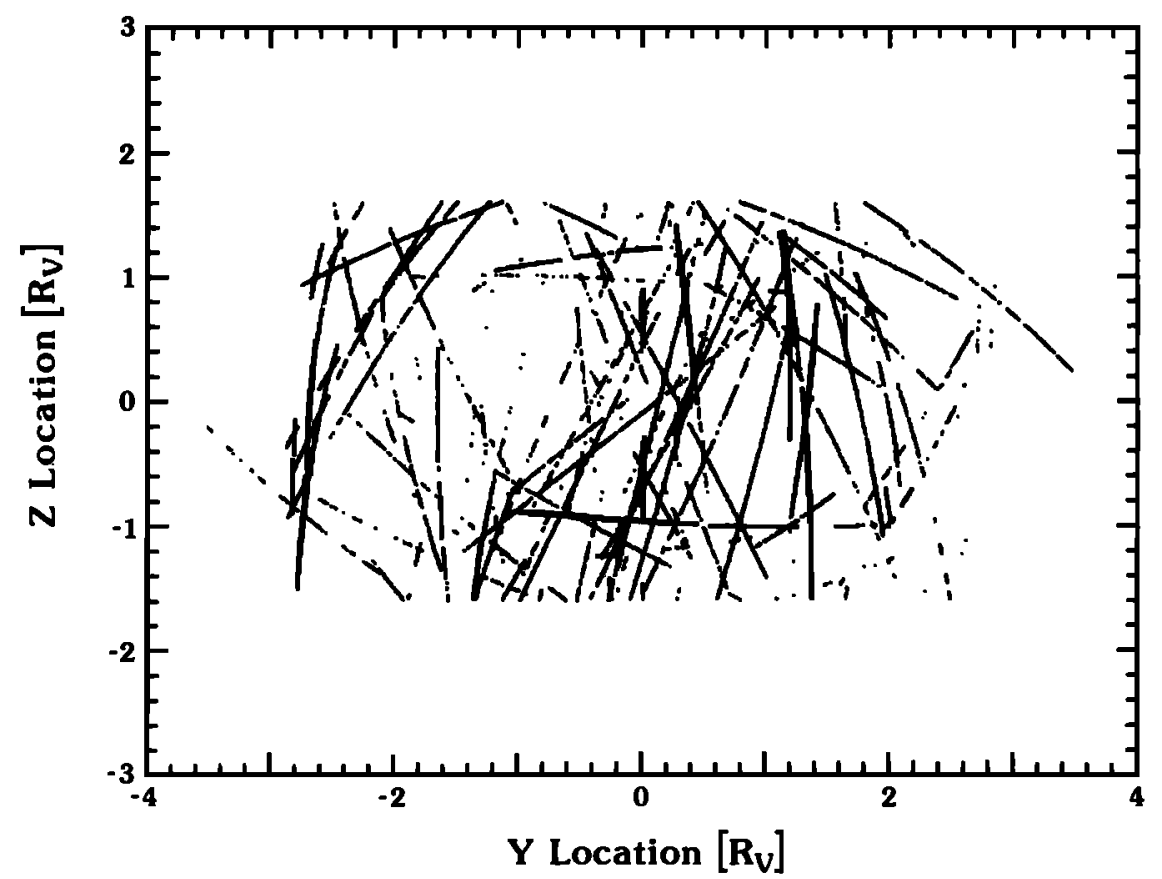

Fig. 2. The orbital coverage of our data set which includes 9423 one-minute averaged magnetic field data points. The data are shown in the $Y-Z$ plane of the cross-flow $(B-v)$ coordinate system in which the upstream magnetic field points along the $+Y$ axis. The data set is truncated at $Z= \pm 1.6 R_{V}$ in order to reduce the effects of a circular tail cross section when the data set is compressed in $Z$.

This choice permits the separation of "east-west" asymmetries in which the two lobes, parallel and antiparallel to the IMF, are different. However, it does not allow the detection of "north-south" differences as might occur, for example, if mass loading is asymmetric as predicted by Cloutier et al. [1974, 1976] and as suggested by the findings of Saunders and Russell [1985] and J. A. Slavin et al. (unpublished manuscript, 1985). There is no choice of coordinate system that will allow us to determine simultaneously both east-west and north-south asymmetries while combining data obtained in different solar wind sectors, since the interaction is inherently three dimensional. However, in order to increase our statistical accuracy, we wish to combine such data. As will become evident in this study, the east-west asymmetry will be very important, and therefore we choose to convert the IMF polarities prior to rotating about the solar wind flow.

The IMF is highly variable, and when the Pioneer Venus spacecraft is probing the Venus tail, it spends little time in the solar wind. When available, any solar wind observations are far removed in time from the periods of the tail observations. Ideally, it would be desirable to have a measure of the IMF direction when Pioneer Venus is within the tail. Saunders and Russell [1986] have established that the cross-tail or $Y$ component is everywhere, on average, parallel to the component of the upstream IMF that is perpendicular to the flow. This is what is expected if there is little reconnection of the field between the two tail lobes. Given these results we can use the direction of the cross-tail component measured on each crossing through the tail to rotate into the desired $B-v$ coordinate system. During each tail crossing, the component of the field in the plane perpendicular to $X$ is measured, and its average direction is used to rotate the coordinate system for the entire crossing so that the average field projection lies along the $B-v$ $+Y$ direction.

Figure 2 shows the orbital coverage of our data set projected onto the $Y-Z$ plane of the $B-v$ coordinate system. All data have been accepted in the full width of observation in the cross-flow, $Y$, direction; however, the data set has been truncated in the $Z$ direction at \pm 1.6 Venus radii $\left(R_{V}\right)$. This truncation ensures that the draping, which is expected to occur somewhat differently as a function of $Z$ in the tail, owing to the variation in the tail width with $Z$, does not mix together the draping patterns from very different portions of the tail. By choosing only the central portion where the tail width is fairly constant and over which draping can be expected to be fairly similar and then by compressing this data set into the two-dimensional $X-Y$ plane, we maximize the statistical accuracy of our derivation of the two-dimensional draping pattern in the tail. Further, our choice of coordinate system has already mixed together north-south asymmetries so that our analysis has already suppressed the vertical gradients in the tail.

Small sections of orbits observed in this figure correspond to portions of the time when the orbit, although continuous in real space, is not continuous in the effective space of the $B-v$ coordinate system. These correspond to times when the IMF variation is large enough that the field points in a reverse cross-flow sense, as will be described shortly. At these times it is necessary to reflect not only the field, but also the location in the tail in the cross-flow coordinates, so that toward and away sectors are again not mixed.

In both panels of Figure 3 the magnitude of the magnetic field in the tail divided by the average magnitude of the magnetic field in the magnetosheath immediately adjacent to the tail, where the PVO pierces the magnetopause on an orbit-byorbit basis, is plotted versus the angle of the field from the cross-flow $Y-Z$ direction toward the positive aberrated $X$ axis. Of course, the magnitude of the magnetic field within the sheath is a function of location as well as the upstream conditions. Along the flanks of the tail from -8 to $-12 R_{V}$, however, the fractional magnetic field strength (compared to upstream) is probably not a strong function of the exact lo- 


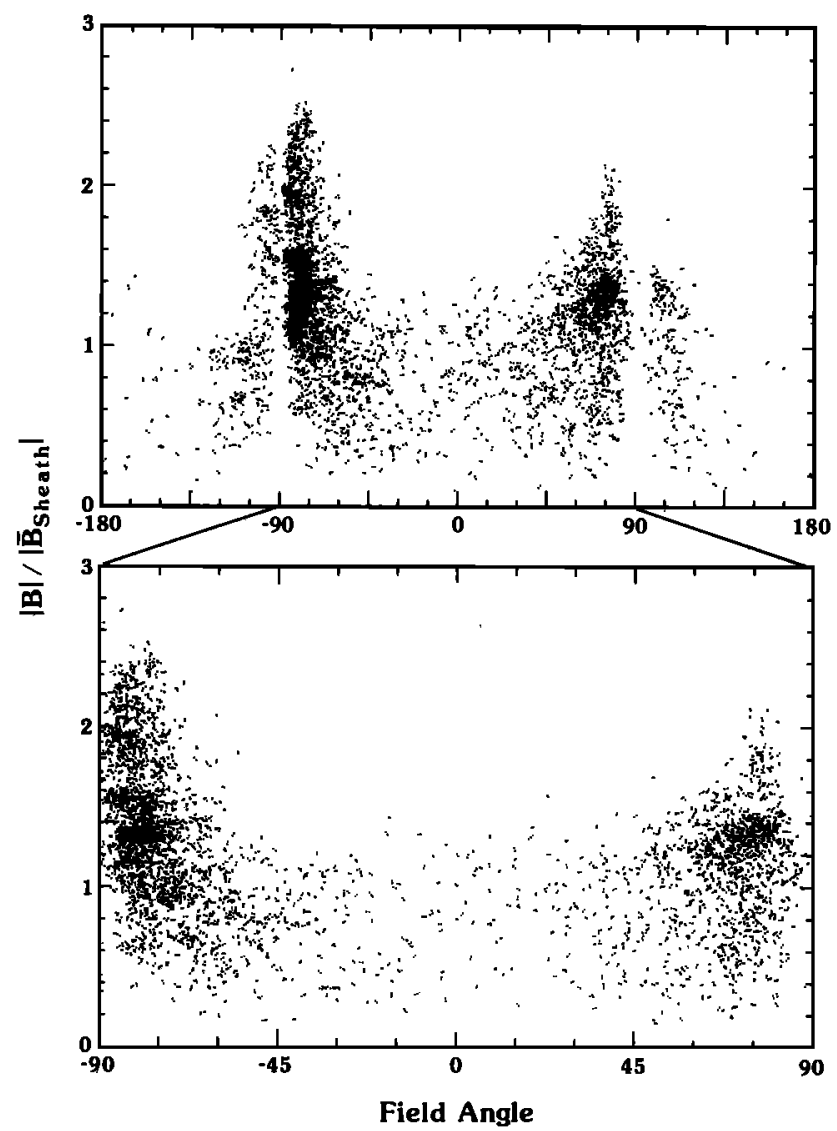

Fig. 3. The magnitude of the magnetic field divided by its average value in the immediately adjacent portion of the magnetosheath, plotted versus the magnetic field angle as measured from the cross-flow $Y-Z$ direction toward the aberrated $X$ axis. The top panel shows a correlation between large relative field strengths and angles near $\pm 90^{\circ}$ and indicates that $79 \%$ of the data lie within these angles. The bottom panel shows the same data set where the remaining $21 \%$ of the data have been folded into the central portion (see text).

cation of the PVO's magnetopause crossings and only serves to introduce scatter into the effect observed in Figure 3. Still, this figure clearly reveals a strong correlation between fields which are close to $\pm 90^{\circ}$ and the larger relative field strengths. We identify these regions where the field is comparatively strong and points basically tailward or Venusward as being the induced lobes of the Venus magnetotail. The term "induced lobes" is used to distinguish these regions from the tail lobes in the terrestrial magnetosphere where the earth's intrinsic dipole field plays a fundamental role. At Venus the draped lobe regions are composed purely of "hung up" interplanetary magnetic field lines.

In contrast to the induced lobes, the magnetic field in the Venus tail current sheet is observed to occur at small angles and at small field strengths. The decrease in the relative field strength may be due to a diamagnetic reduction in the center of the tail consistent with an increase in the plasma density, as is observed in the earth's magnetotail. Exclusions near $\pm 90^{\circ}$ may be due to the purely geometric effect of reduced solid angles near $\pm 90^{\circ}$, or may indicate that the angles in the lobes never truly achieve $\pm 90^{\circ}$ and that the variations in aberration of the tail are smaller than or comparable to the spread in the draping angle in these lobes.

Twenty-one percent of the data in the top panel of the figure are observed to lie outside of $\pm 90^{\circ}$. In order to deter- mine whether this was a property of the tail or caused by the variability of the upstream conditions, the same analysis which yielded this result was repeated on $2701 \mathrm{~min}$ of magnetosheath data taken just outside the Venus tail over the same orbits as in our tail data set. In the magnetosheath this reverse draping, which is indicated by angles outside of $\pm 90^{\circ}$, occurred $16 \%$ of the time. Therefore approximately $16 \%$ out of $21 \%$ of the tail data which lies outside of $\pm 90^{\circ}$ is accounted for simply by the fluctuations in the upstream IMF direction causing reverse draping. The extra $5 \%$ which is still unaccounted for may be due to reconnection or other physical processes. In any case, for the first time, an upper bound has been set on the importance of magnetic reconnection in the Venus magnetotail. Only $\sim 5 \%$ of the observed field in the Venus magnetotail points in a reverse draped sense, and therefore our analysis indicates that magnetic reconnection is probably not an important physical process in the central Venus magnetotail.

The same data set is shown in Figure $3 b$, with the measurements outside of $\pm 90^{\circ}$ "folded" across the $\pm 90^{\circ}$ lines. This folded data set will be used throughout the remainder of this study in order to obtain the most statistically significant results. Folding is reasonable since the observed variation between lobe and current sheet also occurs outside of $\pm 90^{\circ}$ where the $0^{\circ}$ current sheet area is similar to the $\pm 180^{\circ}$ portion. Since three fourths of the data outside of $\pm 90^{\circ}$ can be accounted for by variations in the IMF, truncation of these data would principally lower the statistics of the data set. It is therefore advantageous to fold these data back into the data set by reflecting the data points across $\pm 90^{\circ}$.

A similar plot for orbit 1761 alone is shown in Figure 4. (The magnetometer data for this orbit are displayed in Figure 2 of Saunders and Russell [1986].) The data points have been connected to show the time series in which the data were taken. In motions between the two lobes the spacecraft always encounters the current sheet. That is, there are one or more data points obtained within the current sheet, which are characterized by small angles and small field strengths, in the transition from one lobe to another. The motion of the spacecraft back and forth in the tail is clearly not due to the spacecraft orbital motion ( 24 hours/orbit), since there are many of these comparatively quick (a few minutes) crossings in a single orbit. Rather, some sort of large variations in the tail configuration (perhaps due to the IMF $X$ component) and/or in its location occurs on the time scale of a few 1-min data samples. These variations cause the spacecraft to be alternately in one lobe and then the other, crossing back and forth across the current sheet many times. This temporal variation has previously made it impossible to do a thorough analysis of the average spatial structures of the Venus tail. These large variations, however, are used in a new way in the next section of this study to improve the statistics.

The field angle versus the $B-v Y$ location is shown in Figure 5. Current sheet(s) and lobes are observed at all locations where the tail is encountered, which is consistent with the observations of large variations in most orbits and is exemplified by the plot of orbit 1761. In Figure 5 there is a clear preponderance of either tailward or Venusward pointing fields on each of the two sides of the tail. Magnetic fields near $+90^{\circ}$ occur preferentially on the $-Y$ side of the tail, while fields oriented near $-90^{\circ}$ occur predominantly on the $+Y$ side. In Figure 6 this finding is quantified.

For the purposes of determining the number of induced tail 


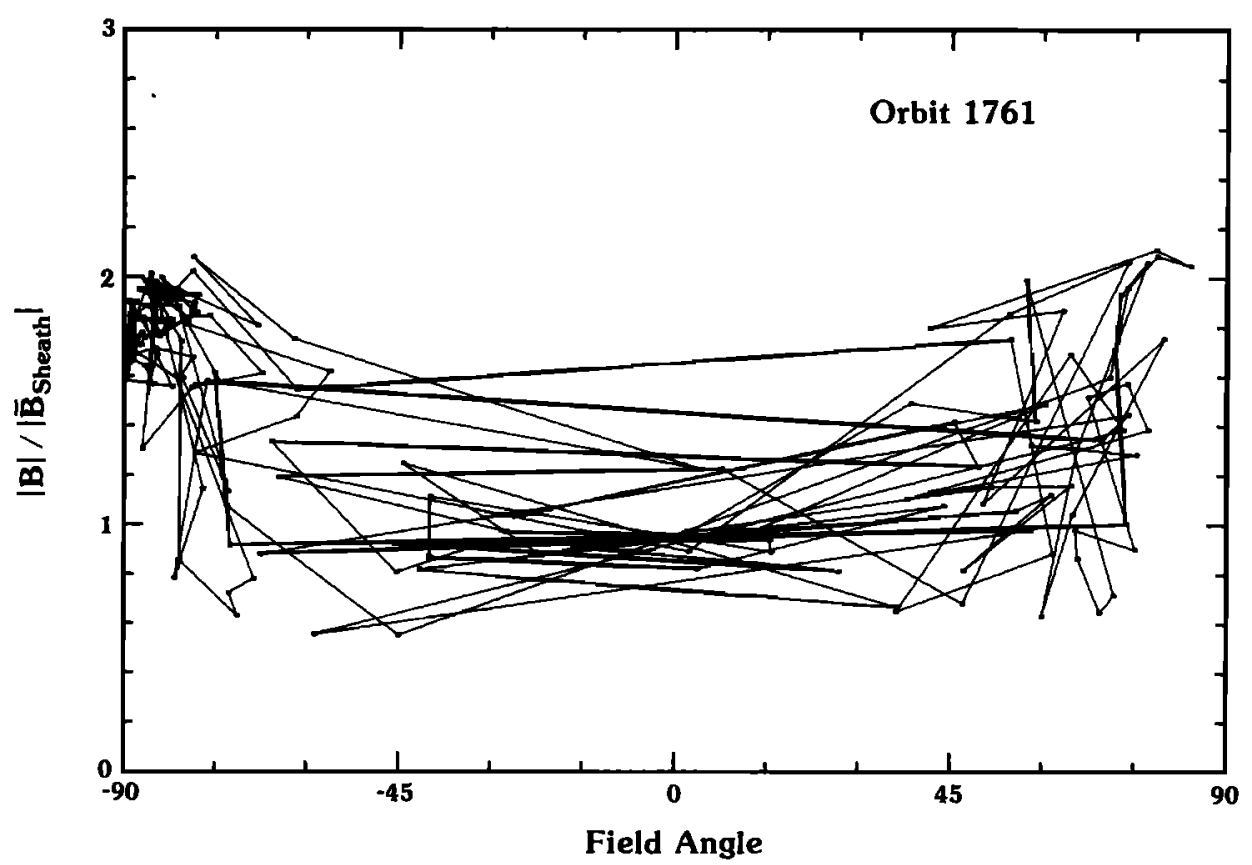

Fig. 4. Similar to Figure 3 except that only data from orbit 1761 are shown. The data points have been connected to show the time series in which the data were taken. The spacecraft traverses the tail many times in an individual orbit. Evidently, the size scales of motions and/or reconfigurations of the tail are much larger than those due to the actual orbital motion. Therefore the data will be much better ordered by a coordinate system which measures locations with respect to the internal tail structures rather than one which measures them in normal spacecraft coordinates.

lobes, we have, somewhat arbitrarily, defined them as consisting of all data points in which (1) the field strength divided by the directly adjacent sheath value (as described previously) is greater than one, and (2) the absolute value of the angle is greater than $60^{\circ}$ (top left and right corners of Figure 4 and the bottom panel of Figure 3). The fraction of the tailward pointing tail lobe is plotted versus the $B-v Y$ location in Figure 6, and two draped lobes are clearly indicated. The number of draped lobes was not something that was assumed in our analysis, but rather it is a result confirmed by our analysis, which merely assumed that magnetic field draping is the important phenomena in the tail. We have repeated this analysis using other lobe cutoff criteria, and all yield the same result.

Figure 4 demonstrated that draped lobes are separated by current sheets, while Figure 6 confirms that there are only two lobes in the Venus magnetotail. Therefore the Venus magnetotail structure is shown to consist of two roughly opposite pointing draped lobes separated by a single current sheet, just

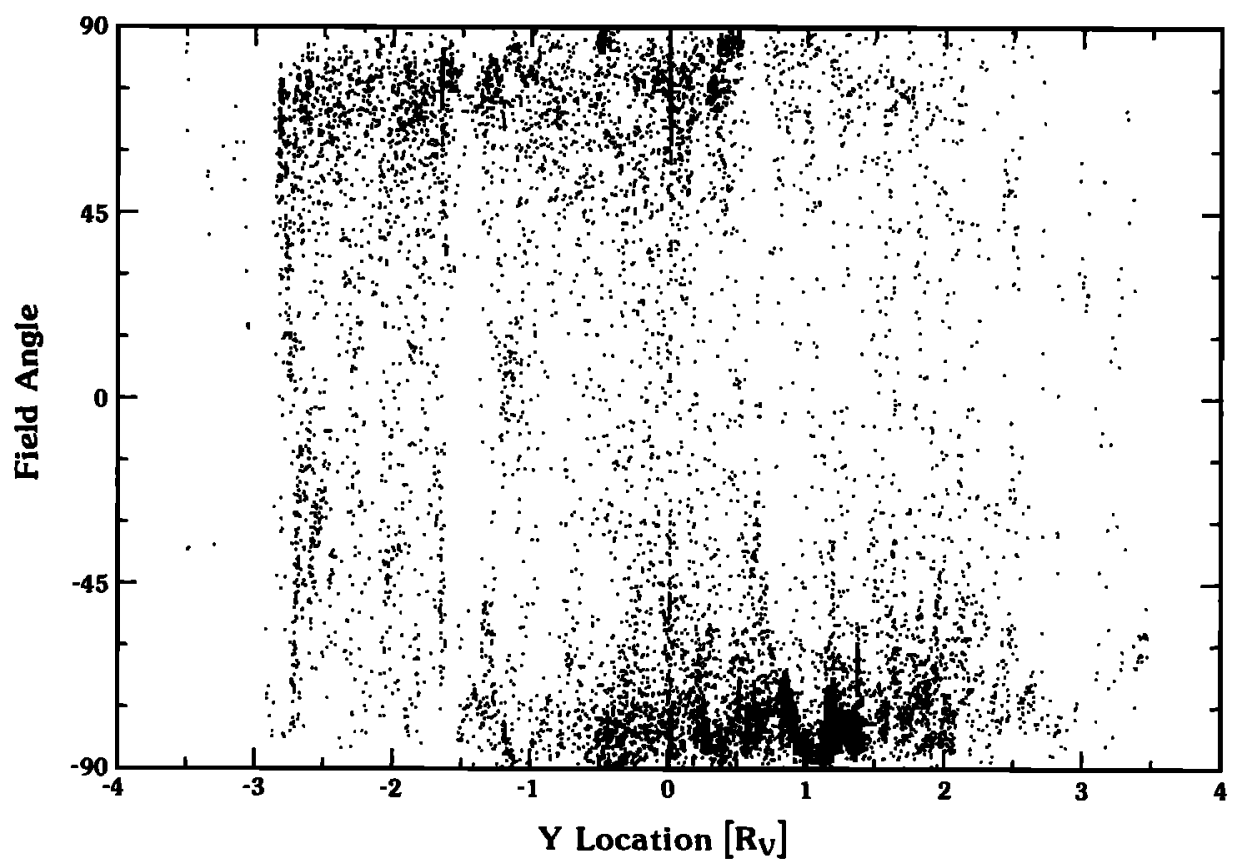

Fig. 5. The magnetic field draping angle versus the $B-v \quad Y$ location. Both direction lobes and current sheet(s) are observed at all locations antisunward of Venus. There are, however, more tailward pointing lobe data on one side of the plot and more Venusward pointing data on the other side. 


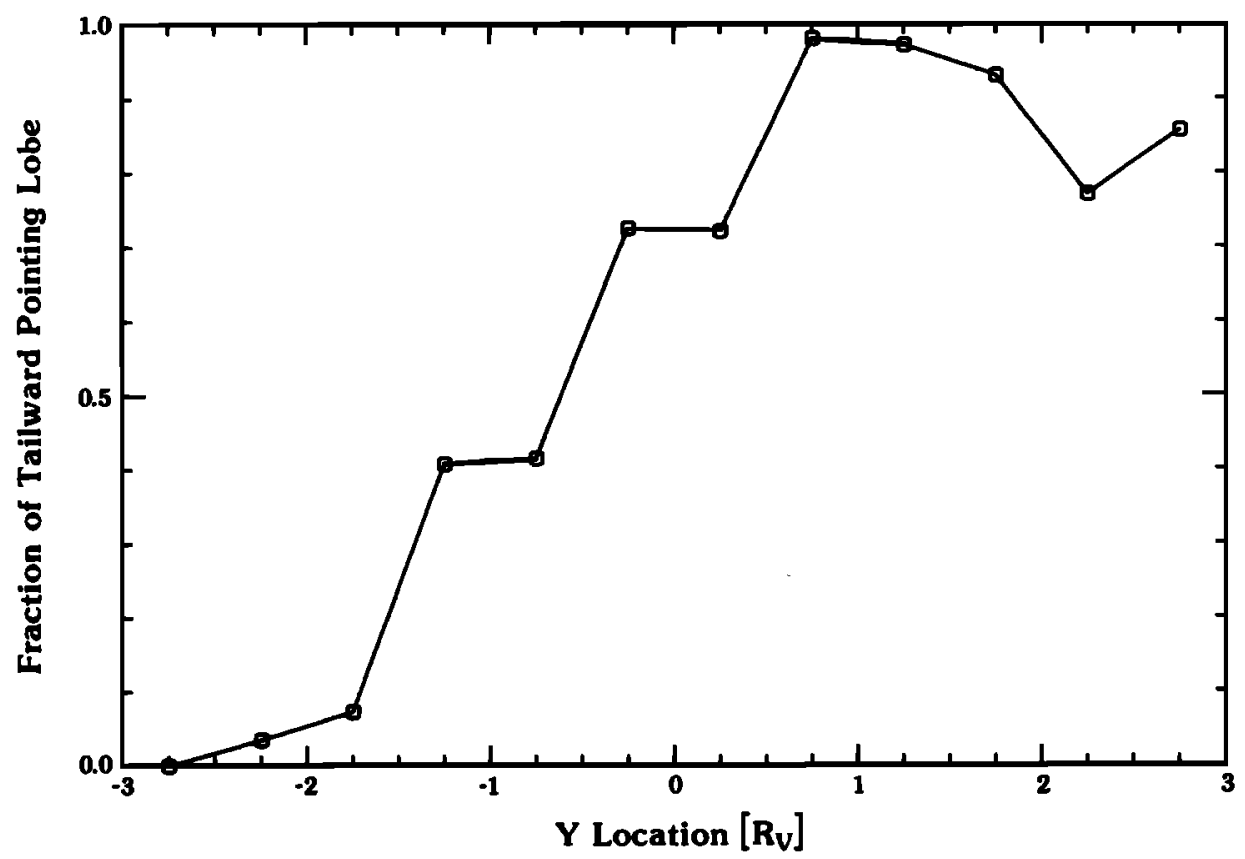

Fig. 6. The fraction of tailward pointing lobe as a function of the $B-v Y$ location. The average tail configuration consists of only two draped lobes which are separated by a single field reversing current sheet.

as we anticipated in Figure 1. The average location of the current sheet center is approximately $-0.5 R_{V}$. This is consistent with the IMF $X$ component mapping into the tail so that, for a normal Parker spiral pattern and an away sector, the magnetic flux in the draped lobe which contains the tailward pointing field is larger than the flux in the draped lobe containing the Venusward pointing magnetic field. In a pressure balance situation the current sheet between these two lobes will tend to move toward the side of the Venusward pointing lobe.

Since we are principally interested in determining the average configuration of the Venus magnetotail, the average field line draping angles in the lobes must be determined. Figure 7 shows the measured average lobe angles determined using the lobe criteria described above for Figure 6. Other, similar lobe criteria have also been tried and yield very similar average angles. These field angles in the two lobes will be used to set the average angle to which the field drapes in the lobes on the distant sides of the tail. The tailward pointing lobe average angle is $-78.4^{\circ}$, while the Venusward average angle is $+73.4^{\circ}$. This asymmetry can also be accounted for quite simply by the mapping of the IMF $X$ component through the magnetosheath and into the tail as shown in Figure 1.

\section{The Average Tall Configuration WITH THE EFFECTS OF FLAPPING REMOVED}

In all previous studies, and thus far in this study, the Venus magnetotail data have been ordered only by the spatial location of the PVO with respect to Venus. Our analysis, however, has indicated that motions and/or reconfigurations of the tail happen far more rapidly than the PVO orbital motion, causing the spacecraft to traverse the tail many times per orbit. In order to advance the understanding of the Venus magnetotail quantitatively, it is necessary to find a coordinate system which better orders these highly variable data by statistically organizing them with respect to the tail features themselves. Since a relation between the diamagnetic re- duction and the field angle has already been demonstrated, it is reasonable to construct a coordinate system in which crossflow locations do not correspond to locations in physical space but rather correspond to locations with respect to the center of the moving or flapping current sheet.

For the data set used in this study, we have empirically determined the tail width to be $5.1 R_{V}$. This was accomplished by determining the fraction of time that each spatial location behind Venus was engulfed within the tail. Since tail motions are large, even the average center location of the tail was apparently within the actual tail only $\sim 97 \%$ of the time, and locations to the sides were within the tail far less. Under these conditions it is not appropriate to simply find the locations which are within the tail $50 \%$ of the time and determine the average width of the tail from these. Rather, the fractional tail coverage behind the planet must be integrated, and the effective tail width calculated from it so that this integrated value equals the tail width multiplied by $100 \%$ (of the time).

We have already shown that the magnetotail is comprised of two draped lobes separated by a cross-tail current sheet and that fields on the $+Y$ side of the tail point tailward, while the fields on the $-Y$ side point Venusward for an away IMF sector draping. Further, since the diamagnetic reduction apparently gives a measure of the average distance from the center of the current sheet, we can now build a coordinate system which is centered on the current sheet and which measures distances from that center. Large variations in the tail configuration and location cause all regions to be sampled at any physical location in the tail. In a statistical data set, such as the one used for this study, the fraction of the total data points which lie within any given angular range gives a direct measure of the relative width of that portion of the tail. The sum of all of these relative widths must, of course, equal the total width of the Venus tail, $5.1 R_{V}$. It is then possible to construct the average configuration of the tail by starting with the average angles derived in each of the two lobes and calculating the thickness of each of the angular bins across the tail.

The basic principle involved in creating such a coordinate 


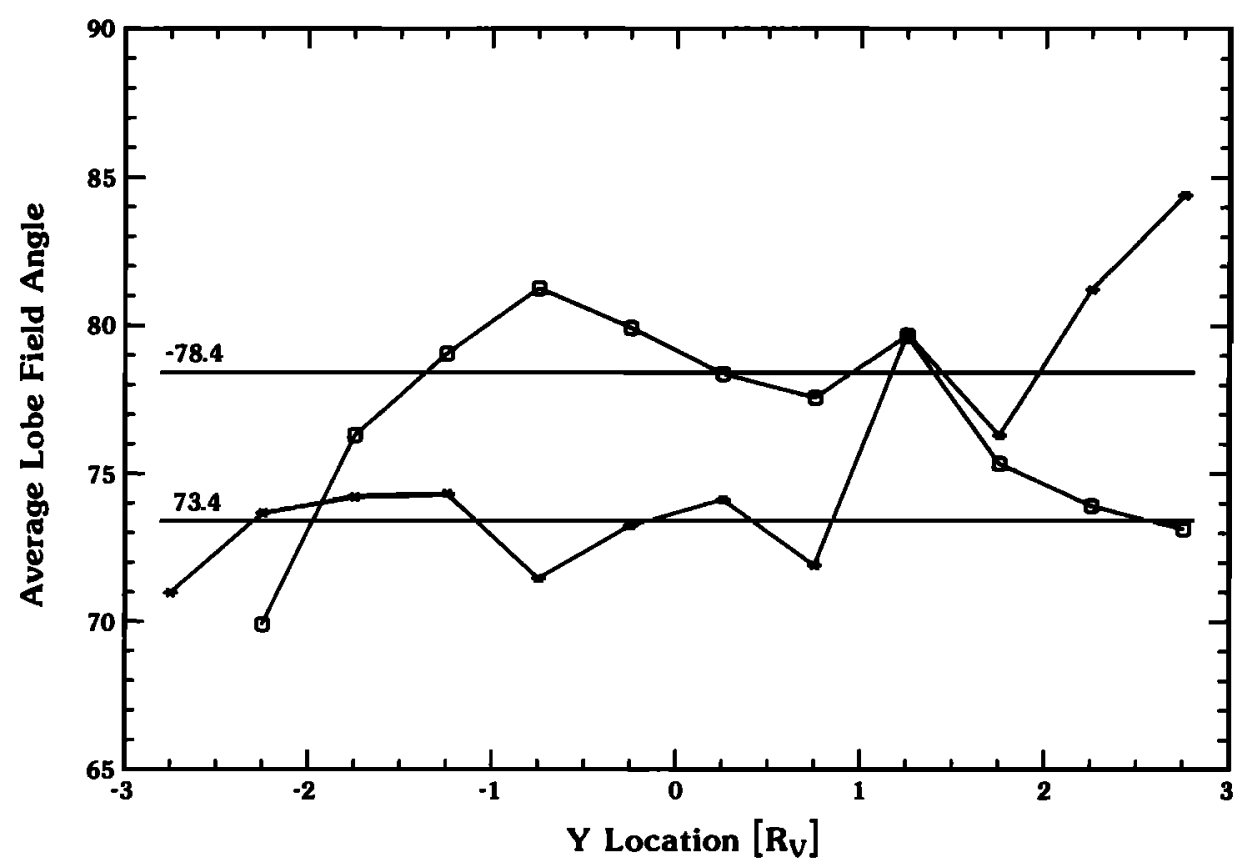

Fig. 7. The average angles of the field line draping in the outer portions of the two lobes. The tailward point lobe value is $-78.4^{\circ}$, while the Venusward lobe is $+73.4^{\circ}$.

system is shown schematically in Figure 8. For simplicity the tail has been divided into only three portions, namely, the $+X$ pointing lobe, the $-X$ pointing lobe, and the current sheet. The lower portion of the figure is a schematic diagram of Figure 5. Since variations of the spacecraft position with respect to the internal structures of the tail are so large, all regions are sampled at every $B-v Y$ location, and the fraction of field in each of the three angle bins gives a direct measure of the thickness of that portion of the tail. In this example the $+X$ lobe, current sheet, and $-X$ lobe are $32 \%, 28 \%$, and $40 \%$ of the tail width, respectively. Since the tail is $5.1 R_{V}$ across, these would correspond to $1.6,1.4$, and $2.1 R_{V}$ for each portion, respectively. This technique is simply extended in our study to measure the thickness of each $1^{\circ}$ angle bin across the tail, and thereby we reconstruct the average tail configuration. The resultant $Y^{*}$ coordinate axis constructed by this technique is parallel to, and shares a Venus centered origin with, the $B-v Y$ axis.

The angle of the magnetic field versus this cross-tail location, $Y^{*}$, is shown in Figure 9. The cross-tail locations of various angles in the tail have been determined as described in the previous figure, while the $X$ axis in these new coordinates is the same as the $X$ axis in the aberrated VSO coordinates. The procedure described above of dividing up and determining the relative amount of data in each $1^{\circ}$ angle bin has been carried out over $\pm 0.5,1.0,1.5$, and $2.0 R_{V}$ in $B-v Y$ from Figure 5. All four of these analyses were carried out independently, and the minimum and maximum angles of the four have been plotted at each $Y^{*}$ location to give the upper and lower bounds displayed as the two light lines in Figure 9. The darker, center line is the average of these four analyses and is adopted for the purposes of this study, hereafter. The very small variation between the center average line and the minimum and maximum lines indicates that it is relatively unimportant over how much of the central portion of the tail the analysis is done. Variations are sufficiently large in tail configuration and location that all portions of the tail are sampled with little orbital prejudice everywhere between \pm 0.5 and $\pm 2.0 R_{V}$. This, then, is the (*) coordinate system in which we can much better measure the average physical features of the Venus tail.

Angles beyond the average angles found in the lobes have been folded into the angles just below the average lobe angles. This is necessary because the analysis fails as the lobe draping angle approaches $\pm 90^{\circ}$, and at angles of $\pm 90^{\circ}$ the field lines
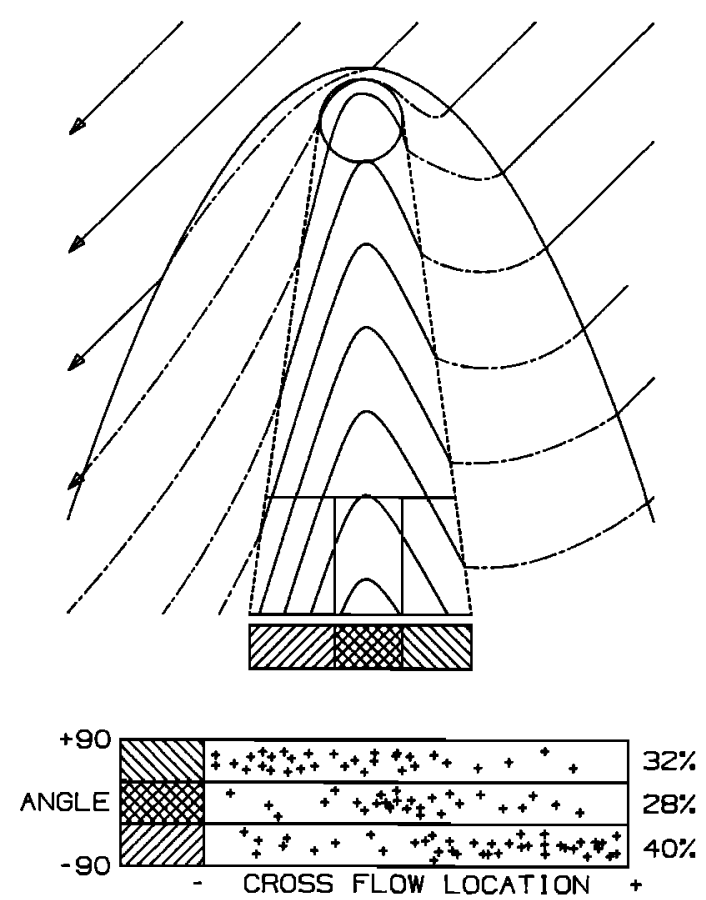

Fig. 8. Schematic diagram of the basic principle used in defining our constructed coordinate system which measures locations with respect to the internal tail structure. The fraction of data points within any range of angles gives the relative thickness of the portion of the tail which encompasses those angles. Combining this information with the total, physical width of the tail gives the spatial scale to the cross-tail coordinate axis. 


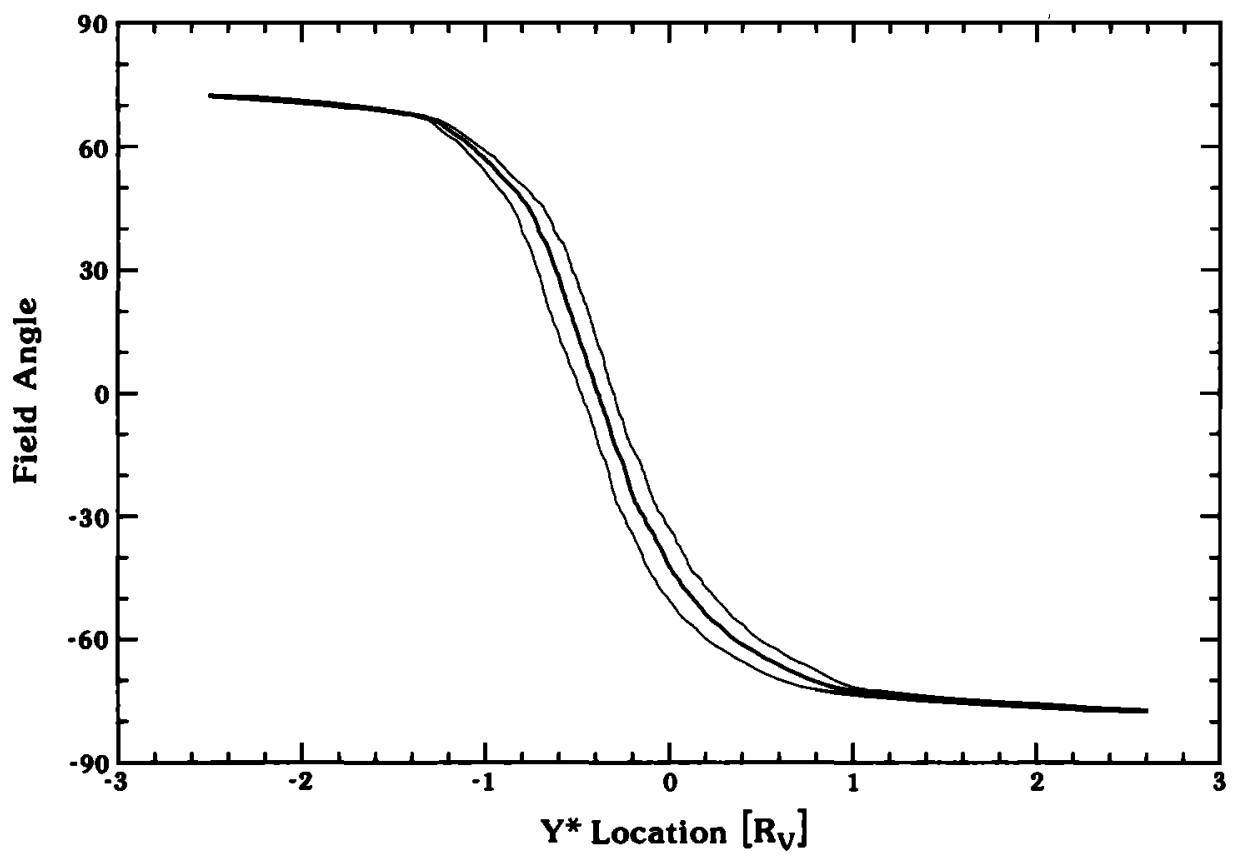

Fig. 9. The angle of the magnetic field plotted versus the $Y^{*}$ location. The two lighter lines are the upper and lower bounds found by carrying out our analysis over $\pm 0.5,1.0,1.5,2.0 R_{V}$. The darker, center line is the average of these and is adopted for the remainder of this study.

would apparently not cross the tail at all. We expect that these infrequently measured large angles are not characteristic of the actual, averaged draping, and probably result from variations in the aberration angle of the tail. Since we are principally interested in the average magnetic field draping in the tail, it is desirable to have the fields come to their average lobe values at the edges of the lobes.

The tailward/Venusward magnetic field, $B_{X}$, is plotted versus $Y^{*}$ in Figure 10 . These are actual measurements over the 9423 data points binned up angle by angle and compared to their $Y^{*}$ locations, and are not calculated from the angles used to derive the $\left(^{*}\right)$ coordinates. This plot, therefore, gives a good independent check of the ability of the $\left(^{*}\right)$ coordinate system to order the data. The two draped lobes are easily discerned in this plot, as is the smooth variation between the two through the current sheet. The zero point in $B_{X}$ once again occurs slightly offset toward the negative side of the tail. The variation displayed has been calculated in an average sense over $X$; the entire data set has been compressed in $X$ so that only variations in the cross-tail direction are determined. Throughout this paper such averages will be indicated with \langle\rangle , so that $B_{X}$ as a function of $Y^{*}$ and averaged over $X$ is

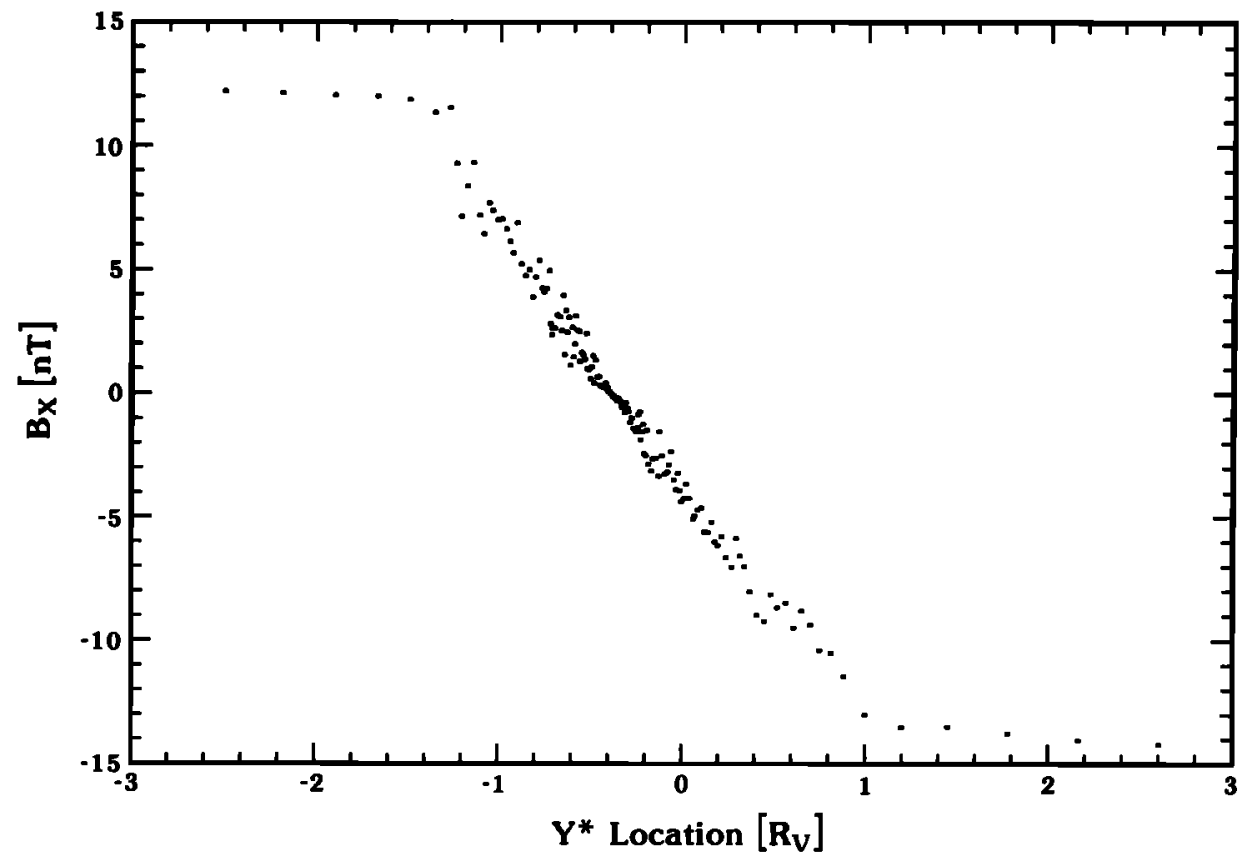

Fig. 10. The actual, measured $B_{X}$ plotted versus $Y^{*}$ in each of the $1^{\circ}$ angle bins. This plot gives a good independent check of the ability of the coordinate system to order the data set. 


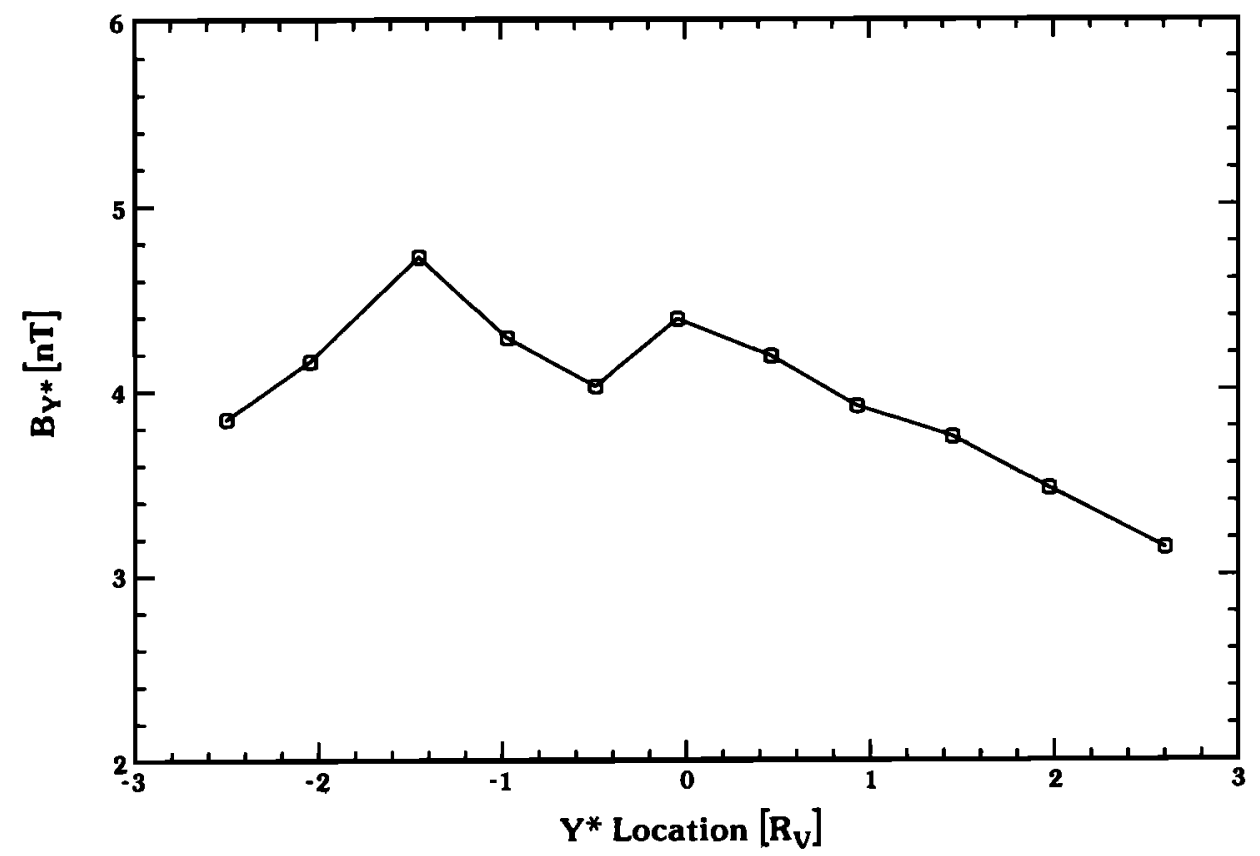

Fig. 11. The measured $B_{Y^{*}}$ plotted versus $Y^{*}$. The variation is calculated in 11 bins across the tail, which vastly improves the statistics.

written $\left\langle B_{X}\left(Y^{*}\right)\right\rangle_{X}$. This average $B_{X}$ as a function of $Y^{*}$ will be used shortly to calculate one of the terms of the current density distribution in the cross-tail current sheet.

A similar plot for the cross-flow magnetic field, $B_{Y^{*}}$ as a function of $Y^{*}$ is displayed in Figure 11. The distribution has been calculated in 11 bins in $Y^{*}$ to improve the statistics. The double-humped variation exhibited in this plot is qualitatively similar to the variation observed in the Fedder simulation of cometary magnetotails [Fedder et al., 1986; J. Phillips, private communication, 1985]. By combining the results in Figures 10 and 11, the field draping in an average sense across the tail is determined. This average draping pattern is shown in Figure

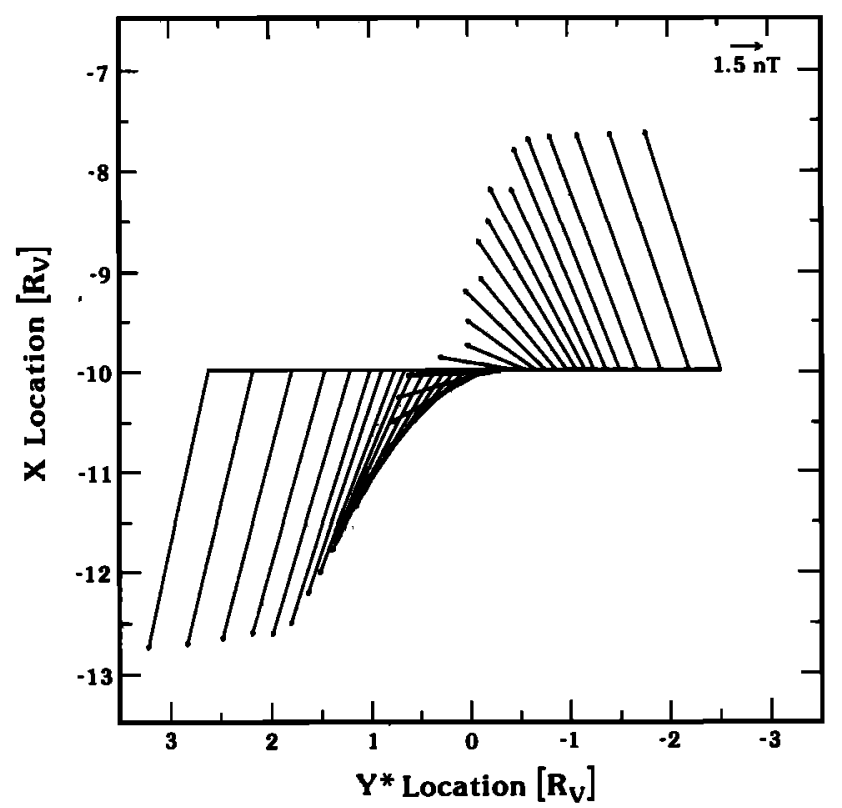

Fig. 12. The average magnetic field vectors determined with our technique as a function of the $\mathrm{Y}^{*}$ location across the tail. The two draped lobes and the smooth rotation through the current sheet between them are all clearly shown.
12. It must be emphasized that these are not magnetic field lines but rather, average field vectors in a crosscut through the tail along $Y^{*}$. Data from -8 to -12 Venus radii have been compressed in $X$ to give this draping pattern. Figure 12 clearly displays the two draped lobes and current sheet in which the field smoothly rotates from one lobe to the other. The tailward side is thicker owing to larger flux content, as well as having a larger tailward field component due to the mapping in of the $X$ component of the IMF, just as we postulated in Figure 1.

Next, we extend the understanding of the field draping in the tail by examining variations of the field in the $X$ direction. Unfortunately, it is only possible to examine the $B_{Y_{*}}$ field variation with $X$ since the $B_{X}$ variation is such a critical function of sampling location in $Y^{*}$. $B_{X}$ varies from about $\pm 13 \mathrm{nT}$ in the lobes to $\theta$ in the center of the current sheet, and, therefore, any orbital bias can strongly affect the determination of the exact variation of the $X$ component. On the other hand, the $B_{Y *}$ component is relatively constant across the tail and is therefore not much affected by orbital bias. In the top panel of Figure 13 we șhow the orbital coverage in $0.5 R_{V}$ bins, where data from -6 to $-12 R_{Y}$ have been included in order to maximize the region covered. The distribution strongly favors data being taken past $-10 R_{V}$ because the PVO orbit apoapsis occurs near $-12 R_{V}$.

The lower panel of Figure 13 displays $B_{Y^{*}}(X)$ along with the best linear fit to this variation. This best fit line equally weights all data points and does not, therefore, overly stress the less significant data bins closer to the planet. The equation for this best fit line for $B_{Y_{*}}$ as a function of $X$, again averaged over $Y^{*}$, is $\left\langle B_{Y *}(X)\right\rangle_{\mathrm{Y} *}=11.53+0.68(X)$ in nanoteslas, where $X$ is in Venus radii. From this variation and from the variation which was shown in Figure 10 the cross-tail current density can be calculated.

The cross-tail current density is numerically evaluated from the curl of the magnetic field according to Ampere's Law. Using the average variation just described, $d\left\langle B_{Y_{w}}\right\rangle_{Y_{m}} / d X=$ 0.61 in the units of nanoteslas/Venus radius from the linear fit 


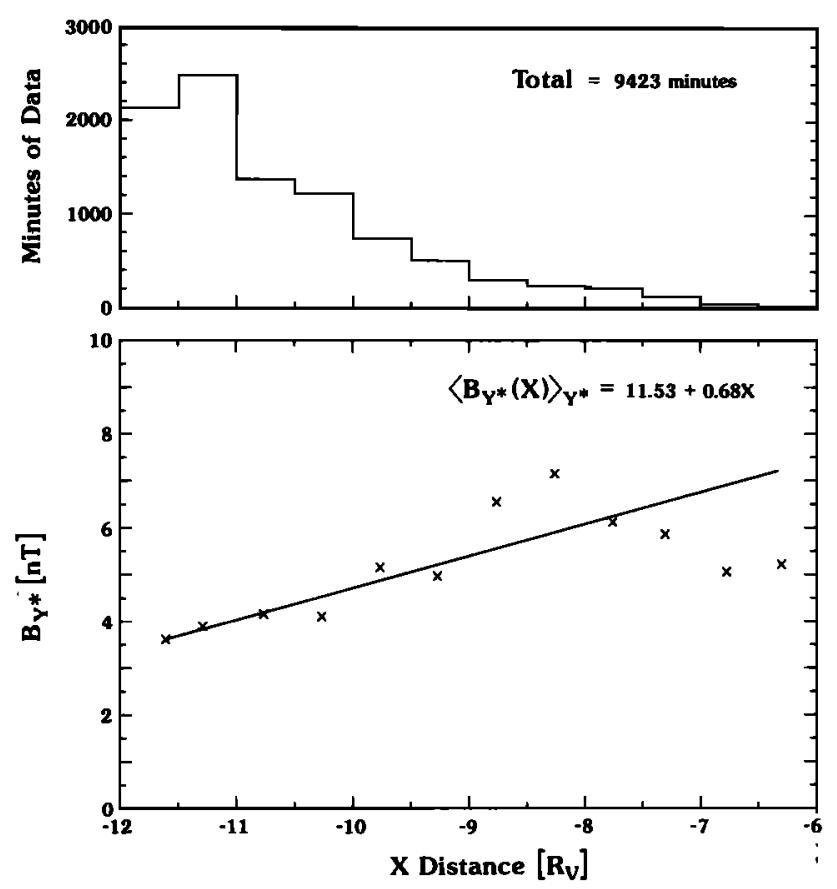

Fig. 13. The previous analysis is extended to examine the variation of the field with downtail, $X$, distance. The top panel shows the orbital coverage of our data set as a function of $X$, while the bottom panel shows the $Y^{*}$-averaged variation of $B_{Y_{*}}(X)$. The best linear fit to this variation is also shown.

to the field variation. This gives a small offset most easily observed in Figure 14 at the two far sides of the tail, while the principal variation in current density through the center is due to the term $d B_{x} / d Y^{*}$ and is calculated from the variation shown in Figure 10. For Figure 14 we have derived the crosstail current density in 11 steps in $Y^{*}$ to smooth out variations which are observed in the derivatives. Clearly, the cross-tail current density is maximized near the center of the tail at $-0.5 R_{V}$ and drops to very small values which correspond primarily to the linear $d B_{Y^{*}} / d X$ term near the edges of the lobes.

From this calculated variation and from the field which was shown in Figure 12, the $\mathbf{J} \times \mathbf{B}$ forces can be calculated in an average sense across the tail. These forces are shown in Figure 15. All forces have a tailward component, and these $-X$ directed forces maximize in the center. Forces on both sides of the tail all point toward the center of the tail. Throughout the side portions of the tail these inward forces are greater than tailward forces. Asymmetries in the side to side distribution of forces are due not only to actual asymmetries in the tail configuration but also, to some extent, to the coarseness of our sampling in $Y^{*}$.

\section{The Inferred Plasma Properties}

The average variations of the magnetic field draping in the Venus magnetotail are not just interesting in their own right, but also contain significant information about the average plasma properties throughout this region. In particular, the $X$ variations of the downtail plasma velocity, $v_{X}(X)$, and acceleration, $a_{X}(X)$, can be directly obtained from the average field variations and the continuity of the tangential electric field. The average plasma density in the current sheet and lobes, and average ion plasma temperature in the tail, on the other hand, can be derived from the calculated plasma acceleration and MHD momentum equation.

The reconstructed $\left(^{*}\right)$ coordinate system developed in this study allows us to represent the steady state, average configuration of the Venus magnetotail. As such, the effects of timevarying upstream conditions have been removed, and the resultant variations determined with our analysis can be treated as though they were derived for constant (average) upstream interplanetary conditions. In this coordinate system the consistent steady state plasma properties of the Venus magnetotail can be calculated approximately from our average magnetic field data. The MHD momentum equation in the two-dimensional $X-Y^{*}$ plane of the field draping is

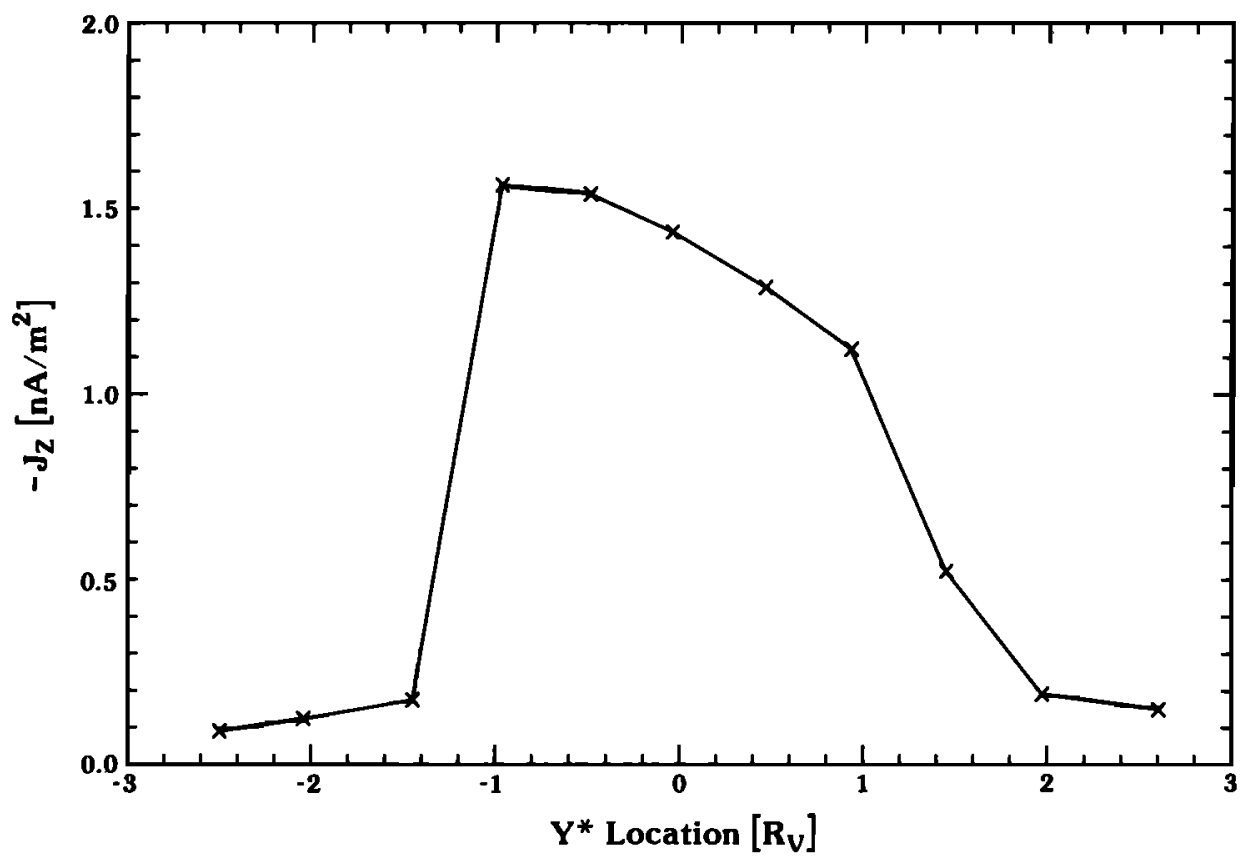

Fig. 14. The cross-tail current density as a function of cross-tail location. Both terms of the curl of the field are included in this calculation. The term $\partial B_{Y *} / \partial X$ gives a small constant offset which is most easily observed at the two edges of the tail, while the primary component through the center of the tail is from $\partial B_{X} / \partial Y^{*}$. 


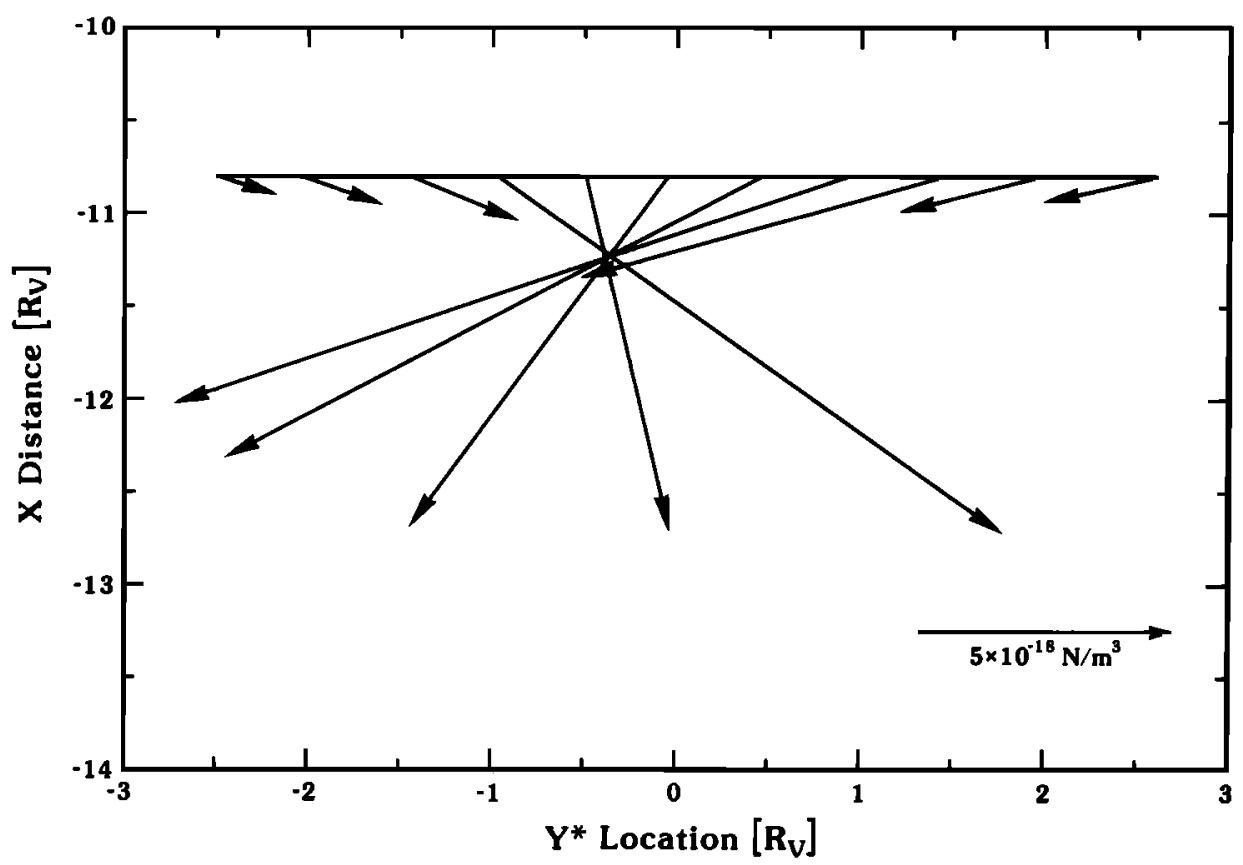

Fig. 15. The derived $\mathbf{J} \times \mathbf{B}$ forces as a function of $Y^{*}$ across the tail. The $X$-directed forces all point tailward and maximize in the tail center, while the $Y^{*}$-directed forces point inward on both sides. These forces are the important electromagnetic term of the momentum equation with which we examine the consistent plasma properties of the Venus magnetotail.

$$
\begin{gathered}
(\mathbf{J} \times \mathbf{B})_{X}=-J_{Z} B_{Y}=\rho\left(v_{X} \frac{\partial}{\partial X} v_{X}+v_{Y} \frac{\partial}{\partial Y} v_{X}\right) \\
(\mathbf{J} \times \mathbf{B})_{Y}=J_{Z} B_{X}=\rho\left(v_{X} \frac{\partial}{\partial X} v_{Y}+v_{Y} \frac{\partial}{\partial Y} v_{Y}\right)+\frac{\partial P}{\partial Y}
\end{gathered}
$$

The pressure gradient term has been dropped in (1a) beçause there is no boundary confining the plasma in the $-X$ direction, as there are in the $\pm Y$ directions, and the plasma is therefore free to flow downtail. For simplicity, we have dropped the (*) from all $Y$ terms in (1) and throughout this section; however, it should be understood that all equations and discussions in this section refer to the average, steady state configuration described by the statistical $X-Y^{*}$ coordinate system.

If (1) the plasma pressure in the current sheet greatly dominates the magnetic field pressure $(\beta \gg 1),(2)$ the opposite condition holds in the lobes $(\beta \ll 1)$, and (3) $Y$ directed accelerations are not too large, then the $Y$-directed $\mathbf{J} \times \mathbf{B}$ force in (1b) will be primarily balanced by the gradient in the plasma pressure alone rather than the inertial forces. In this case, virtually all plasma flow will be directed downtail, and $V_{Y}(X, Y) \approx 0$. Therefore (1a) simplifies to include only the first of the two spatial derivative terms. This sort of tail configuration is expected if ion pickup (e.g., $\mathrm{O}^{+}$) is an important physical process in the extended dayside ionosphere or if solar wind plasma flowing near to the stagnation point on the dayside of Venus flows around the obstacle and into the magnetotail. This limit of the equation will be explored quantitatively in this section.

Unfortunately, even the large statistical data set used in this study is insufficient to accurately determine the full twodimensional magnetic field variations. Instead, we have derived the average variations of the tail field in directions perpendicular to these averages. For example, we derive the variations of the field with $Y$ in an average sense in $X$. The task at hand now is to use these average variations to derive the average tail plasma properties. In general, the average value of a product of two functions does not equal the product of the average values of these functions. However, when one or both of the functions is approximately constant over the direction of averaging, these two types of averages are approximately equal.

The $Y$-averaged variation of the downtail velocity, $\left\langle v_{X}(X)\right\rangle_{Y}$, is derived from the continuity of the tangential electric field or, equivalently, the conservation of magnetic flux. The tangential electric field is

$$
E_{Z}=-v_{X} B_{Y}+v_{Y} B_{X}
$$

and is constant and perpendicular to the two-dimensional plane of the magnetic field draping. $E_{Z}$ is roughly equal to the product of the $X$ component of the magnetosheath plasma velocity and the magnetosheath magnetic field $Y$ component. The average magnetic field crossing the flanks of the tail is, from Figure 11, $3.5 \mathrm{nT}$. The average flow speed in the magnetosheath along the flanks of the magnetopause, tailward of $\sim 4 R_{v}$, is $>90 \%$ of the upstream solar wind speed and is directly along streamlines which are very nearly pointing in the $-X$ direction [Spreiter and Stahara, 1980]. For this study we will assume the tailward magnetosheath velocity near to the magnetopause from -8 to $-12 R_{V}$ to be $440 \mathrm{~km} / \mathrm{s} . E_{Z}$, therefore, is simply $440 \times 3.5=1.54[\mathrm{mV} / \mathrm{m}]$.

In the limit studied here, $v_{Y} \approx 0$ and (2) becomes simply $-v_{X} B_{Y}=1.54[\mathrm{mV} / \mathrm{m}] . B_{Y}$ is approximately constant as a function of the $Y$ location, varying by only $\sim \pm 15 \%$. Therefore, to within the accuracy of this derivation, the $v_{X}$ and $B_{Y}$ terms of this equation can be averaged separately over $Y$. This procedure yields the equation for the average tailward velocity

$$
\left\langle v_{X}(X)\right\rangle_{Y}=\frac{-1540}{\left\langle B_{Y}(X)\right\rangle_{Y}}=\frac{-1540}{11.53+0.68 X}
$$




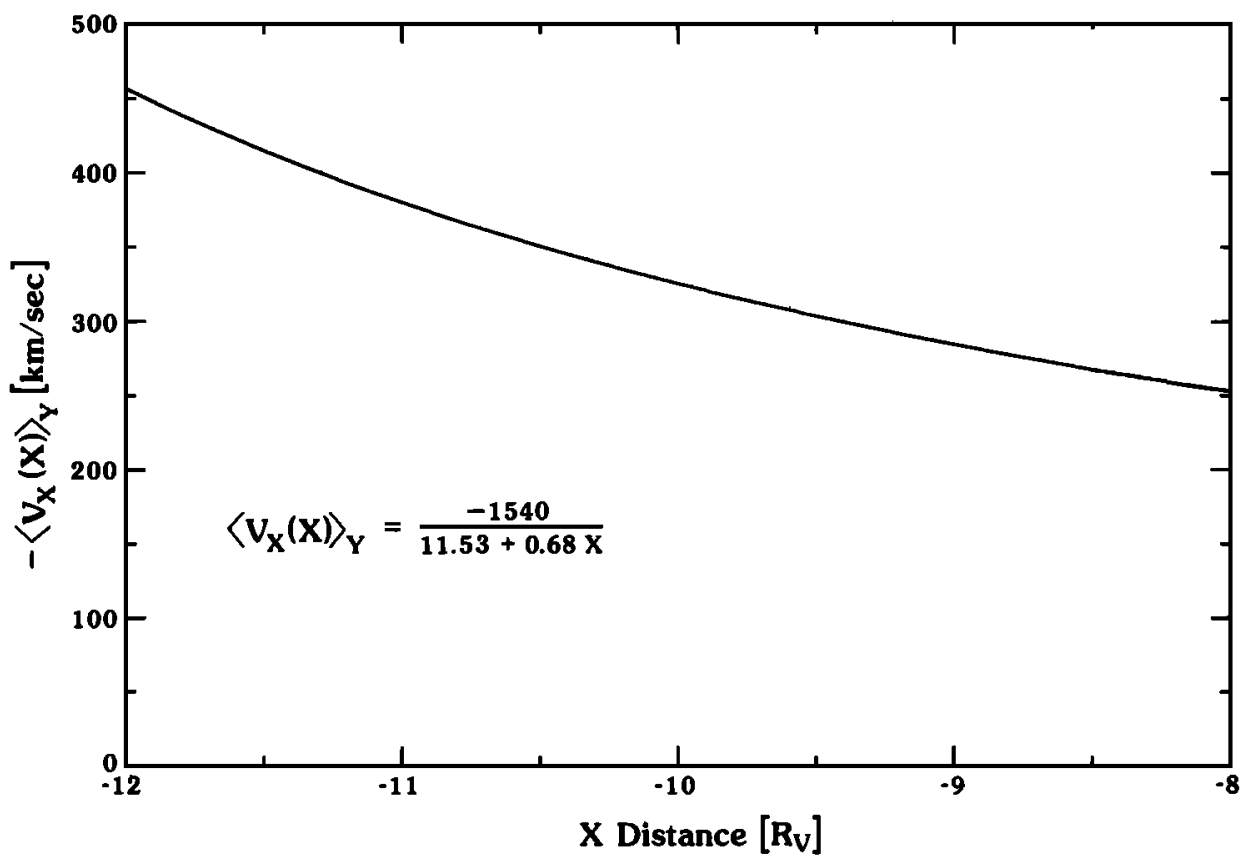

Fig. 16. The $Y$-averaged tailward plasma velocity, $\left\langle v_{X}(X)\right\rangle_{Y}$, as a function of downtail distance. Equation (3) describes this variation quantitatively. Venusward of $-11.8 R_{v}$, the flow is slower than the nominal solar wind speed, while beyond this distance the field lines are catching up with the nominally straight IMF configuration.

where the velocity is given in $\mathrm{km} / \mathrm{s}$, and $X$ is in $R_{V}$. This function is plotted in Figure 16 from -8 to $-12 R_{V}$, which is the range over which it is reasonably reliably determined.

Extrapolation of (3) outside the range over which our data set extends is problematic. The qualitative result, however, that tailward plasma motion near Venus is quite slow compared to the solar wind speed is well determined. The deep tail region from -8 to $-12 R_{V}$ is shown in this study to be an interesting transition region. Tailward of $-8 R_{V}$ the average plasma velocity increases from about $-250 \mathrm{~km} / \mathrm{s}$ to the solar wind speed of $-440 \mathrm{~km} / \mathrm{s}$ at $\sim-11.8 R_{V}$, and therefore the draped field throughout this portion of the tail continues to fall further behind the IMF. At $-12 R_{V}$ the average plasma velocity is greater than the solar wind speed, and the field in this portion of the tail is in the process of catching up with the comparatively straight IMF configuration.

The steady state plasma acceleration is simply the spatial derivative $v_{X} \partial v_{X} / \partial X$. The $Y$-averaged spatial acceleration, $\left\langle v_{X} \partial v_{X} / \partial X\right\rangle_{Y}$, cannot be uniquely determined from (3); however, the product of the average terms in the acceleration gives

$$
\left\langle v_{X}\right\rangle_{Y} \partial\left\langle v_{X}\right\rangle_{Y} / \partial X=\frac{-267}{(11.53+0.68 X)^{3}}
$$

in $\mathrm{km} / \mathrm{s}^{2}$, where $X$ is in $R_{V}$. This spatial acceleration is shown in Figure 17 from -8 to $-12 R_{V}$.

The precise plasma density distribution and temperature cannot be uniquely calculated from the average velocity and acceleration derived above. The approximate values of these quantities, however, can be calculated from the variations and the momentum equation. In the limit described in this study, (1) becomes

$$
\begin{gathered}
-J_{z} B_{Y}=\rho\left(v_{X} \partial v_{X} / \partial X\right) \\
J_{Z} B_{X}=\partial P / \partial Y
\end{gathered}
$$

Insertion of the average field, currents, and plasma acceleration into $(5 a)$ yields

$$
\rho \simeq \frac{\left\langle-J_{Z}\right\rangle_{X}\left\langle B_{Y}\right\rangle_{X}}{\left\langle v_{X}\right\rangle_{Y} \partial\left\langle v_{X}\right\rangle_{Y} / \partial X}
$$

By averaging the denominator of (6) over $X$, the remaining $Y$ variation of the numerator then determines the gross $Y$ variation of the density. Of course, the plasma acceleration also varies as a function of $Y$, but, since the average plasma velocity is similar to the sheath velocity in this portion of the tail, the acceleration is probably not a strong function of $Y$. In addition, it must be remembered that these are only rough calculations and that all terms cannot be fully treated with the average variation information gathered in this study. In the draped lobes the derived mass density is then $\sim 1.2 \times 10^{-22}$ $\mathrm{kg} / \mathrm{m}^{3}$, while the average derived mass density in the current sheet is $\sim 1.6 \times 10^{-21} \mathrm{~kg} / \mathrm{m}^{3}$. These values are equivalent to densities of approximately 0.07 and 0.9 protons $/ \mathrm{cm}^{3}$, respectively. If the plasma in the Venus tail is composed of $\mathrm{O}^{+}$, then the equivalent densities are 0.005 and $0.06 / \mathrm{cm}^{3}$.

These average values of the plasma density are in good agreement with the lack of observations of plasma in the Venus magnetotail by the PVO plasma analyzer [Intriligator et al., 1980]. This instrument was principally designed for solar wind observations and does not have the sensitivity to measure plasmas with densities of $\$ 1 / \mathrm{cm}^{3}$ (M. A. Saunders et al., unpublished manuscript, 1985). Since our derived average plasma densities are lower than this cutoff, we would predict that the Venus tail would generally be indicated by a "plasma dropout" of the plasma analyzer. This is actually observed to be the case [Mihalov and Barnes, 1982]. When present, these observations usually show both $\mathrm{O}^{+}$and proton peaks in the spectra, but the spectra are almost never well enough formed to actually derive moments from. Even where it is possible to derive moments, these would represent unusual plasma conditions in the tail (perhaps high density and low flow speed), while our derived plasma properties are consistent with the average, steady state configuration of the tail.

The typical plasma temperature in the Venus tail can be 


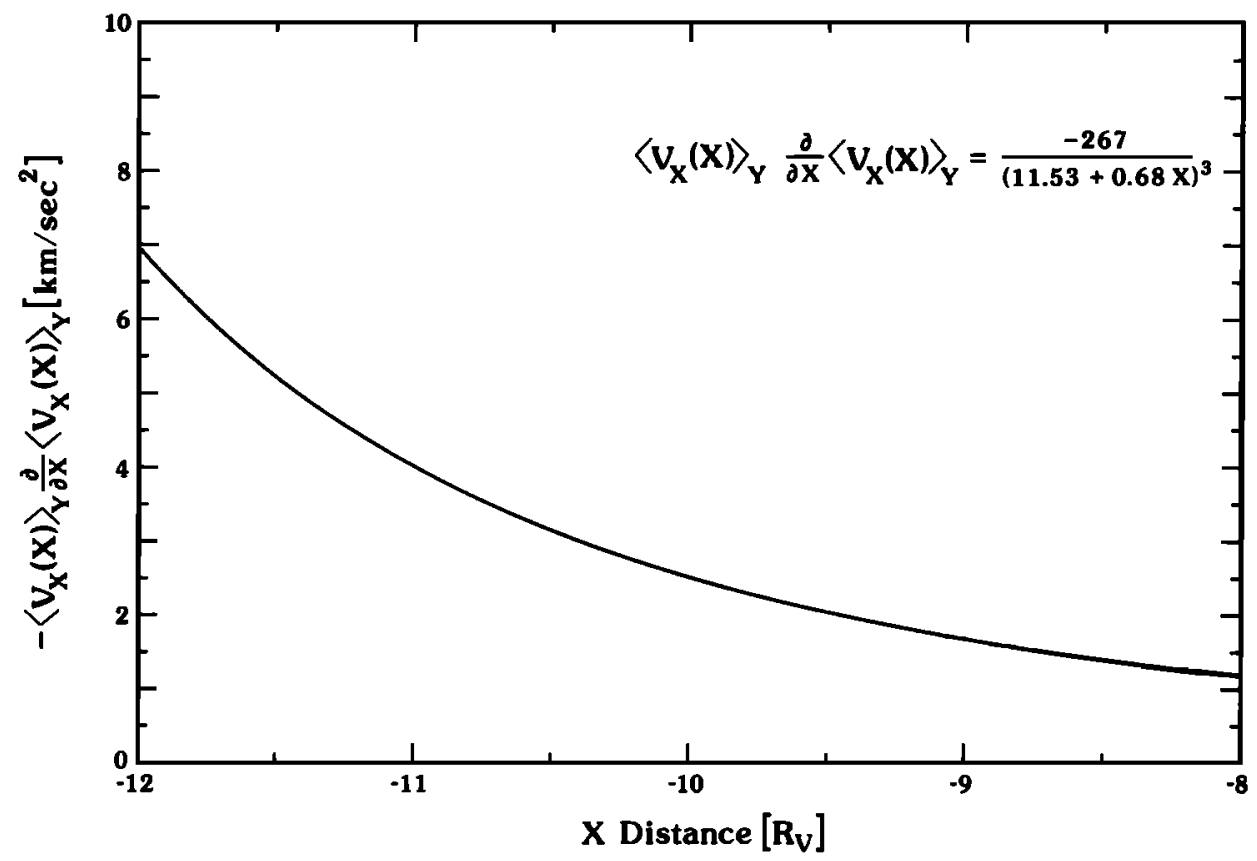

Fig. 17. The $Y$-averaged plasma acceleration $\left\langle v_{X}\right\rangle_{Y} \partial\left\langle v_{X}\right\rangle_{Y} / \partial X$ as a function of downtail distance. Equation (4) describes this variation quantitatively.

derived from $(5 b)$ by using the variation in the density which was just determined. We derive only the approximate plasma temperature averaged over this entire portion of the tail, since the variations derived in this study are insufficient to determine gradients in the temperature. Averaging all terms in $(5 b)$ and replacing $\partial P / \partial Y$ with $k T(\Delta n / \Delta Y)$, we solve for the temperature which gives

$$
T \simeq \frac{\left\langle\left\langle J_{Z}\right\rangle_{X}\right\rangle_{Y}\left\langle\left\langle B_{X}\right\rangle_{X}\right\rangle_{Y}}{K} \frac{\Delta Y}{\Delta n}
$$

where $K$ is Boltzmann's constant and $\Delta n$ and $\Delta Y$ are typical variations in density and $Y$ location between the lobes and current sheet. Equation (7) was numerically evaluated using $\left\langle\left\langle B_{X}\right\rangle_{X}\right\rangle_{Y}=10 \mathrm{nT}$ and the derived variation in the density between the draped lobes and the current sheet. If the tail is assumed to consist entirely of hydrogen, then the proton temperature is of the order of $6 \times 10^{6} \mathrm{~K}$. If, on the other hand, the plasma is principally formed by $\mathrm{O}^{+}$, then the ion temperature is of the order of $9 \times 10^{7} \mathrm{~K}$. These values represent upper bounds only, since we have assumed that the electron pressure is negligible in this final portion of the derivation. If the electron temperature is comparable to or greater than the ion temperature, these values would be much reduced. In addition, successive steps in this derivation have required successively greater assumptions, and therefore these derived temperatures are the least well determined of our plasma parameters. We principally intend that they be used only as rough estimates. These values, however, are not so high as to be impossible to achieve in an induced magnetotail where ion pickup may be important. $\mathrm{O}^{+}$picked up by the unimpeded solar wind can have temperatures as high as $\sim 2 \times 10^{8} \mathrm{~K}$ when they are picked up with an initial perpendicular (thermal) velocity of the typical solar wind speed $(440 \mathrm{~km} / \mathrm{s})$.

Using the approximate plasma parameters derived above, the lobe and current sheet $\beta^{s}$ can be calculated. The plasma $\beta$ in the lobe is $\sim 0.08$, which is consistent with the difference in the magnetic flux in the two lobes moving the current sheet from side to side and with our assumption of low $\beta$ lobes in the above derivation. The $\beta$ in the current sheet, on the other hand, is $\sim 12$, which is consistent with our assumption of a high $\beta$ current sheet in the derivation of these plasma parameters. The calculated gyroradii of protons and $\mathrm{O}^{+}$ions calculated from these plasma moments are $\sim 0.1$ and $\sim 1.5 R_{V}$ in the current sheet, respectively, and only $\sim 30 \%$ of that in the lobes. Since these size scales are appreciably smaller than the width of the tail, our use of the fluid momentum equation in this derivation is vindicated.

The average plasma properties derived in this study can be used to determine the approximate average mass flux down the Venus tail, which also represents an upper bound on the mass loss rate of Venus. For this rough calculation we assume that the current sheet and lobes each take up one third of the cross-sectional area of the tail, which we assume to be circular and $5 R_{V}$ in diameter. At the average downtail distance of data in our data set $\left(-10.8 R_{V}\right)$, (3) gives $\left\langle v_{X}\right\rangle_{Y}=-368 \mathrm{~km} / \mathrm{s}$. Multiplying this velocity by the sum of the current sheet and lobe densities times their respective cross-sectional areas, we calculate a downtail mass flux of $\sim 1 \times 10^{26} \mathrm{amu} / \mathrm{s}$ or $\sim 6$ $\times 10^{24} \mathrm{O}^{+} / \mathrm{s}$. If all of the material in the Venus magnetotail is of planetary origin, then this value represents the approximate Venus mass loss rate. In any case, $\sim 1 \times 10^{26} \mathrm{amu} / \mathrm{s}$ represents an upper bound for the mass loss rate of the Venus atmosphere through tail formation.

\section{SUMMARY}

This study began with the schematic diagram of the solar wind/IMF interaction with Venus displayed in Figure 1. We postulated that the IMF $X$ component could have an important effect on the internal structure of the draped magnetotail. In particular, variations of the $X$ component could cause the flapping of the current sheet from side to side within the tail consistent with the magnetic pressure of the varying quantities of magnetic flux in the two lobes. We developed this study in three sections.

In section 2 we demonstrated that magnetometer data ordered by spatial location is extremely variable. Both lobes and 
the current sheet can be found at all spatial locations within the tail, although there is a preference for the tailward pointing lobe on the $+Y$ side and the Venusward pointing lobe on the $-Y$ side. The average separation between the two lobes was shown to be offset toward the lobe which contains less magnetic flux. This observation, along with the high variability observed, confirmed our postulated importance of the IMF $\boldsymbol{X}$ component. Clearly, what was needed to further advance the quantitative study of the Venus tail was to find a coordinate system which measures locations with respect to the internal tail structures themselves.

In section 2 we also showed that magnetic reconnection is a small effect in the central Venus tail and that the magnetic field strength in the tail, referenced to the average magnetosheath value immediately adjacent to the tail, at the points in each orbit where the PVO pierces the magnetopause, is strongly correlated with the magnetic field draping angle. In the lobes the field points roughly tailward and Venusward, and the relative field strength is large. In the lobe separating current sheet, on the other hand, the field points roughly across the tail and the relative field strength is comparatively small. We used this correlation to construct a coordinate system which statistically locates data with respect to the tail current sheet itself.

In section 3 we actually constructed this coordinate system and measured the average variations of the field components for the first time. The $B_{X}$ component was demonstrated to smoothly vary from strongly Venusward on the far $-Y$ side of the tail, through zero in the center of the current sheet, to strongly tailward on the far $+Y$ side of the tail. The $B_{Y *}$ component, on the other hand, showed a two-humped distribution across the tail and decreased fairly linearly with distance from the planet. From these variations we drew the average draping pattern in the deep Venus tail in Figure 12 and derived the average cross-tail current density distribution. Knowledge of the average magnetic fields and self-consistent currents made it possible to calculate the electromagnetic $\mathbf{J} \times \mathbf{B}$ forces and examine the average consistent plasma properties of the tail.

In section 4 we used the continuity of the tangential electric field and found field variations to derive the average tailward velocity and acceleration as functions of downtail distance. The tailward velocity was shown to vary from $\sim-250 \mathrm{~km} / \mathrm{s}$ at $-8 R_{V}$ to $\sim-470 \mathrm{~km} / \mathrm{s}$ at $-12 R_{V}$. From the derived accelerations, the calculated $\mathbf{J} \times \mathbf{B}$ forces, and the MHD momentum equation, we calculated the approximate plasma densities in the current sheet and lobes and very approximate average plasma temperature. The current sheet density was shown to be $\sim 0.9 p^{+} / \mathrm{cm}^{3}\left(0.06 \mathrm{O}^{+} / \mathrm{cm}^{3}\right)$, while the lobe density was approximately $15 \%$ of that. These densities were shown to be consistent with the general lack of PVO plasma observations in the tail. If the tail plasma is composed purely of hydrogen, then we calculate that an average, approximate, maximum ion temperature is $\sim 6 \times 10^{6} \mathrm{~K}$, while if it is composed essentially of oxygen, this temperature is $\sim 9 \times 10^{7} \mathrm{~K}$. Finally, we calculated the mass flux in the tail, which is $\sim 1$ $\times 10^{26} \mathrm{amu} / \mathrm{s}$. This value represents an upper bound for the mass loss rate of the Venus atmosphere through tail formation.

While this study derives many previously unknown average quantities of the deep Venus magnetotail, and sets, for the first time, the average magnetic field draping pattern, selfconsistent currents and forces, and approximate inferred properties of this region, it should not be thought of as an end to the study of the deep Venus magnetotail. Rather, a powerful analysis tool has been developed for examining the detailed physics of this region by referencing the location of not just magnetic field data but all PVO data, with respect to the internal tail structures themselves. Finally, our method may be very valuable in examining the magnetotails of other mass loading obstacles in the solar wind, such as comets, and the deep (reconnected) terrestrial magnetotail, since the orientation of the upstream IMF should also have a strong influence on these regions.

Acknowledgments. We gratefully acknowledge valuable discussions with D. N. Baker, R. C. Elphic, J. G. Luhmann, W. I. Newman, J. L. Phillips, G. L. Siscoe, and D. T. Young. We also gratefully acknowledge valuable constructive critiques from the two referees: J. R. Spreiter and R. P. Lepping. The magnetic field data used in this study and work done at the IGPP, UCLA, were supported under NASA contract NAS2-9491. One of the authors (H.E.S.) was supported by the Office of Naval Research, while another (M.A.S.) was supported by an ESA Research Fellowship. The principal author and work done at Los Alamos were supported by Los Alamos National Laboratory under the auspices of the United States Department of Energy.

The Editor thanks R. P. Lepping and J. R. Spreiter for their assistance in evaluating this paper.

\section{REFERENCES}

Alexander, C. J., and C. T. Russell, Solar cycle dependence of the location of the Venus bow shock, Geophys. Res. Lett., 12, 369, 1985. Alfven, H., On the theory of comet tails, Tellus, 9, 92, 1957.

Bieber, J. W., and E. C. Stone, Energetic electron bursts in the magnetopause electron layer and in interplanetary space, in Magnetospheric Boundary Layers, edited by B. Battrick, pp. 131-135, European Space Agency, Paris, 1979.

Cloutier, P. A., Solar wind interaction with planetary ionospheres, in Solar wind interactions with planets Mercury, Venus and Mars, edited by N. F. Ness, NASA SP-397, pp. 111-119, 1976.

Cloutier, P. A., R. E. Dariell, and D. M. Butler, Atmospheric ion wakes of Venus and Mars in the solar wind, Planet. Space Sci., 22, 967-990, 1974.

Dolginov, S. S., L. N. Zhuzgov, V. A. Sharova, and V. B. Buzin, Magnetic field and magnetosphere of the planet Venus, Cosmic Res., 16, 657, 1978.

Fedder, J. A., J. G. Lyon, J. H. Guiliani, Jr., MHD simulation of the solar wind interaction with comets-Predictions for GiacobiniZinner, Eos Trans. $A G U, 67,17,1986$.

Intriligator, D. S., and F. L. Scarf, Wave-particle interactions in the Venus wake and tail, J. Geophys. Res., 89, 47, 1984.

Intriligator, D. S., J. H. Wolfe, and J. D. Mihalov, The Pioneer Venus Orbiter plasma analyzer experiment, IEEE Trans. Geosci. Remote Sensing, GE-19, $1,39,1980$.

Luhmann, J. G., C. T. Russell, J. R. Spreiter, and S. S. Stahara, Evidence for mass-loading of the Venus magnetosheath, Adv. Space Res., 5(4), 307, 1985.

Mihalov, J. D., and A. Barnes, Evidence for the acceleration of ionospheric $\mathrm{O}^{+}$in the magnetosheath of Venus, Geophys. Res. Lett., 8 , $1277,1981$.

Mihalov, J. D., and A. Barnes, The distant interplanetary wake of Venus: Plasma observations of Pioneer Venus, J. Geophys. Res., 87, 9045, 1982.

Russell, C. T., The magnetosphere of Venus: Evidence for a boundary layer and a magnetotail, Geophys. Res. Lett., 3, 589, 1976.

Russell, C. T., R. C. Elphic, J. G. Luhmann, and J. A. Slavin, On the search for an intrinsic magnetic field at Venus, Proc. Lunar Planet. Sci. Conf., 1lth, 1896, 1980a.

Russell, C. T., R. C. Snare, J. D. Means, and R. C. Elphic, Pioneer Venus Orbiter fluxgate magnetometer, IEEE Trans., GE-18, 32, $1980 b$.

Russell, C. T., J. G. Luhmann, R. C. Elphic, and F. L. Scarf, The distant bow shock and magnetotail of Venus: Magnetic field and plasma wave observations, Geophys. Res. Lett., 8, 843, 1981. 
Russell, C. T., M. A. Saunders, and J. G. Luhmann, Mass-loading and the formation of the Venus tail, Adv. Space Res., 5, 177-184, 1985.

Saunders, M. A., and C. T. Russell, Average dimension and magnetic structure of the distant Venus magnetotail, J. Geophys. Res., in press, 1986.

Slavin, J. A., E. J. Smith, and D. S. Intriligator, A comparative study of distant magnetotail structure at Venus and Earth, Geophys. Res. Lett., II, 1074, 1984.

Spreiter, J. R., and S. S. Stahara, Solar wind flow past Venus: Theory and comparisons, J. Geophys. Res., 85, 7715, 1980.
D. J. McComas, MCC0955289, Los Alamos National Laboratory, ESS-8, M.S. D438, Los Alamos, NM 87454.

M. A. Saunders, The Blackett Laboratory, Imperial College of Science and Technology, London SW7 2BZ, England.

H. E. Spence and C. T. Russell, Institute of Geophysics and Planetary Physics, University of California, Los Angeles, CA 90024.

\section{(Received November 4, 1985; revised March 3, 1986;} accepted March 3, 1986.) 